\title{
BOUNDARY PLASMA TRANSPORT STUDIES ON TFTR
}

\author{
CFFTP G-91112
}

November 1991

\author{
C.S. Pitcher, P.C. Stangeby \\ Canadlan Fusion Fuels Technology Project
}

\section{DISCLAIMER}

This report was prepared as an account of work sponsored by an agency of the United States Government. Neither the United States Government nor any agency thereof, nor any of their employees, makes any warranty, express or implied, or assumes any legal liability or responsibility for the accuracy, completeness, or usefulness of any information, apparatus, product, or process disclosed, or represents that its use would not infringe privately owned rights. Reference herein to any specific commercial product, process, or service by trade name, trademark, manufacturer, or otherwise does not necessarily constitute or imply its endorsement, recommendation, or favoring by the United States Government or any agency thereof. The views and opinions of authors expressed herein do not necessarily state or reflect those of the United States Government or any agency thereof. 
DOE/ER/54091-1

\title{
BOUNIDARY PLASMA TRANSPORT STUDIES ON TFTR
}

\author{
Final Report \\ September 1990-September 1991 \\ C.S. Pitcher and P.C. Stangeby \\ Canadian Fusion Fuels Technology Project \\ 2700 Lakeshore Road West \\ Mississauga, Ontario, Canada \\ L5J $1 \mathrm{~K} 3$
}

November 1991

Prepared for the U.S. Department of Energy Under Grant Number DE-FG05-90ER54091 


\section{Boundary Plasma Transport Studies on TFTR}

\section{Executive Summary}

In this report we summarize the experimental and theoretical investigations supported under the DOE Transport Initiative Contract (\#DE-FG05-90ER54091, contract period Sept 151990 - Sept 14 1991) on the subject of Boundary Plasma Transport Studies on TFTR.

This work can be logically separated into two areas of investigations and therefore the report included here is divided into two parts. First, in Section I, detailed boundary plasma data obtained from TFTR in an Ohmic density scan has been compared with results from the LIM Impurity Production and Transport Code. Second, in Section II, experimental results taken with the newly-purchased (specifically for this Initiative) intensified CCD camera are presented, which, after comparison with simple modelling, reveals a new and important effect that has implications for limiter and divertor plate design.

The following summarizes the main points from these studies:

\section{(I) Detailed Comparison of LIM Code Results with TFTR Ohmic Discharges}

(1) The experimental results are consistent with physical sputtering by deuteron and carbon ions being the dominant impurity release mechanism, provided that an enhancement $\eta$ in the normal incidence yields is allowed for, $\eta \sim 2$.

(2) At low density the carbon self-sputtering yield $\eta Y_{C}$ approaches unity and thus the discharge density is maintained primarily by the carbon influx.

(3) The energy radiated per carbon atom $E_{r a d}$ is constant with density but higher than that predicted using the LIM code.

(4) The carbon particle confinernent time $\tau_{p}$ decreases with density as $\tau_{p} \propto<n_{e}>^{-1}$, dernonstrating the increased screening by the plasma boundary as density increases. 
(5) The central $Z_{\text {eff }}$ is controlled to a large degree by the effective sputtering yield at the limiter $Y \equiv \Gamma_{C} / \Gamma_{D}$, which in turn is a function of the edge temperature $T_{c}(a)$ and the sputtering yield enhancement $\eta$.

(6) At low density, significant quantities of impurities produced at the limiter can spread along the magnetic field a sufficient distance that the effective source for the central plasma is as if it were poloidally uniform. At higher density, impurity ion transport is quite localized, with the typical ion trajectory being confined close to the limiter.

(7) Over most of the density range a significant part of the limiter sink action for impurities is primarily due to cross-field diffusion directly onto the limiter surface. This behaviour is significantly different than would occur for a rail limiter, for example, where the parallel sink action would be dominant.

(8) The TFTR limiter geometry, because it is optimized to reduce surface power density during high power operation and thus has a surface almost tangent to the magnetic field, tends to be more effective at contaminating the central plasma than a rail limiter since sputtered impurities are directed radially inward.

(9) The observation at low density of high particle confinement times $\tau_{p}$ despite long SOL e-folding distances at the outside mid-plane can be explained by a spatial variation of the cross-field diffusion coefficient; small on the high-field side of the plasma and large on the low-field side and with a radial variation, larger in the SOL, smaller in the confined plasma.

\section{(II) Particle and Power Fluxes to Surfaces for a Glancing Magnetic Field}

(1) The plasma flow pattern to the limiter is observed to be very different than at first expected. It is clear that the usual assumption that the particle flux is reduced by the cosine of the angle $\theta$ between the surface normal and the magnetic field is incorrect. That is, the flux density on the surface appears to be independent of field angle for most of the TFTR limiter.

(2) When $|90-\theta|<3^{\circ}$ the parallel-field flux to a surface is reduced to the point where cross-field flux becomes significant and even dominant.

\section{Executive Summary 2}


(3) These results are in apparent contradiction to recent results from DIII where the cosine dependence of the power flux is observed. Further work is required to reconcile this discrepancy.

(4) Because of the importance of the cross-field flux to the limiter surface, the wetted area of the limiter is between $50 \%$ and $100 \%$ larger than expected assuming simple parallel flow and full shadowing by adjacent bays. This implies that the average limiter power loading is lower than expected during high power operation.

(5) It has in the past been assumed in the design of limiters and divertor plates that the particle and power flux incident on the surface can be reduced to arbitrary low values by inclining the structure to produce a glancing magnetic field. This study has shown that there is a lower limit beyond which the particle flux cannot be reduced further. This regime is coincident with that envisioned for future reactors, although such effects have not been taken into account in their design.

\section{Publications}

Three publications have directly resulted from this research:

C S Pitcher, P C Stangeby, R V Budny, C E Bush, J D Elder, K W Hill, S J Kilpatrick, D M Manos, S S Medley, A T Ramsey, J F Schivell and M Ulrickson, "The Effect of Density on Boundan Plasma Behaviour in TFTR", Proceedings of the 18th European Conference on Controlled Fusion and Plasma Physics, Berlin, June 1991, 15C III, 145.

(2) C S Pitcher, P C Stangeby, R V Budny, C E Bush, J D Elder, S J Kilpatrick, D M Manos, S S Medley, A T Ramsey, J F Schivell and M Ulrickson, "The Effect of the Boundary Plasma on TFTR Ohmic Discharges", to be published in Nuclear Fusion (1992).

(3) C S Pitcher, J D Elder, S J Kilpatrick, D M Manos, S S Medley, D K Owens, A T Ramsey, P C Stangeby and M Ulrickson, "Particle and Power Fluxes to Surfaces for a Glancing Magnetic Field", Abstract Submitted to 10th International Conference on Plasma-Surface Interactions in Controlled Fusion Devices, March 30 - April 3, 1992, Monterey, California, USA. 


\title{
(I) Detailed Comparison of LIM Code Results with TFTR Ohmic Discharges
}

\author{
P C Stangeby, C S Pitcher* and J D Elder \\ University of Toronto, Canada
}

* Canadian Fusion Fuels Technology Project

\section{Introduction}

In this report we compare detailed experimental results obtained from TFTR in Ohmic discharges with predictions from the LIM impurity production and transport code.

\section{Experiment}

TFTR has a major radius that is varied typically in the range $R_{0}=2.40 \mathrm{~m}$ to $R_{0}=2.60 \mathrm{~m}$ and a plasma cross-section which is essentially circular with corresponding minor radii of $\mathrm{a}=0.75 \mathrm{~m}$ to $\mathrm{a}=0.95 \mathrm{~m}$. The plasma is limited by a toroidal inner bumper limiter composed of discrete graphite tiles covering an area of $\sim 22 \mathrm{~m}^{2}$, Fig. 1 . The toroidal limiter has a periodic structure in the toroidal direction which repeats at each bay. There are 20 such bays equi-spaced around the torus, corresponding to the 20 toroidal field coils. The limiter shape is such that it produces a maximum plasma interaction in the middle of each bay. Because of the convoluted shape of the limiter, although the nominal graphite area is $\sim 22 \mathrm{~m}^{2}$, the area of the limiter which is in direct contact with the plasma (i.e. "wetted") is $\sim 10 \mathrm{~m}^{2}$.

In the present study, neither the neutral beam injectors nor the ICRF heating was employed. In addition to the core profile diagnostics on the tokamak, TFTR has a number of edge-specific diagnostics, including a reciprocating Langmuir probe located approximately $37 \mathrm{~cm}$ above the outside midplane, a poloidal array of interference filter/telescopes which measure the poloidal $D_{\alpha}$ and $C \|(657.8 \mathrm{~nm})$ distributions at the centre of a bay [1] and a CCD camera plasma viewing system which measures both the poloidal and toroidal distributions of emission from the low ionization states of deuterium, helium and carbon [2]. These states are localized close to the point of the limiterplasma interaction and thus, in general, penetrate no further than $10 \mathrm{~cm}$ inside the last 
closed magnetic flux surface (LCFS). As is typical in most discharges in TFTR [3], the oxygen and metal levels in the plasma are low, except at the highest densities where oxygen can be important. These discharges, however, followed shortly after a boronization procedure which reduces the oxygen level even at high density. Also, because of the low total input power, these discharges did not suffer from carbon blooms [4].

The spectral intensities of the low ionization states can be converted to particle influxes using theoretical photon efficiencies [5], and the local electron temperature as measured with the Langmuir probe.

In the sequence of discharges compared with LIM the following were maintained constant, the major radius $\left(R_{0}=2.45 \mathrm{~m}\right)$, the minor radius $(a=0.80 \mathrm{~m})$, the plasma current $\left(I_{p}=1.4 M A\right)$, the Ohmic power input $\left(P_{\mathrm{\Omega}}=1 M W\right)$ and the toroidal magnetic field $\left(B_{T}=4 T\right)$. The discharge conditions were varied by changing the density through deuterium gas puffing. The range of (volume-averaged) density extended from a lower limit of $\left\langle n_{e}\right\rangle \sim 1 \times 10^{19} \mathrm{~m}^{-3}$ to a high density disruptive limit of $\left\langle n_{e}\right\rangle \sim 4 \times 10^{19} \mathrm{~m}^{-3}$. Across this range, the edge plasma conditions $n_{e}(a), T_{e}(a)$ changed significantly and this is manifested by corresponding changes in the central conditions, as discussed in the next section.

\section{Basic Experimental Results to be Compared with LIM}

Figure 2 gives the boundary plasma conditions as functions of the volume-averaged density $\left\langle n_{e}\right\rangle$. These data were taken from successive discharges which had reached steady state conditions iasting several seconds. In general, measurements of

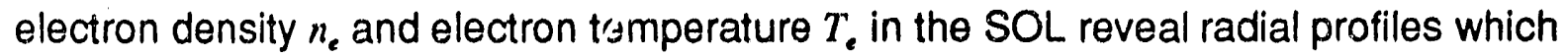
are well-approximated by exponential functions characterized by e-folding distances for density $\lambda_{n}$ and temperature $\lambda_{r}$. The values of density and temperature, $n_{e}(a)$ and $T_{e}(a)$, at the LCFS are taken directly from the probe measurements using the abrupt decrease in floating potential as the probe crosses the LCFS as a means of fixing the position of the LCFS [6]. This position agrees with detailed magnetic calculations to within $\sim 1 \mathrm{~cm}$.

The edge density $n_{e}(a)$ and temperature $T_{e}(a)$ are anti-correlated: at low volume-average density the temperature is high $T_{e}(a) \sim 60 \mathrm{eV}$ and the edge density is low, 
$n_{e}(a) \sim 2 \times 10^{18} \mathrm{~m}^{-3}$, while at high density the temperature is low $T_{e}(a) \sim 25 \mathrm{eV}$ and the edge density is high $n_{e}(a) \sim 1.3 \times 10^{19} \mathrm{~m}^{-3}$. The edge density follows a roughly linear relationship with the volume-average density, $n_{e}(a) \sim \frac{1}{3}<n_{e}>$.

Throughout most of the density range, the e-folding distance for electron temperature $\lambda_{T}$ is significantly longer, i.e. $>10 \mathrm{~cm}$, than the e-folding distance for density, $\lambda_{n} \sim 4 \mathrm{~cm}$. Only at the lowest density are they approximately equal, i.e. $\lambda_{n} \sim \lambda_{r} \sim 6 \mathrm{~cm}$. These lengths are measured at the poloidal position of the reciprocating probe and thus, inferred SOL e-folding distances at the limiter, for example, must take into account the expansion of the flux surfaces from the probe at the outside mid-plane to the limiter at the inside midplane (expansion factor 1.6).

The absolute influxes of deuterium and carbon are obtained from the spectral intensities of the $D_{\alpha}(656.1 \mathrm{~nm})$ and the $C \|(657.8 \mathrm{~nm})$ lines. In the case of the deuterium influx, it is assumed that deuterium recycles from the graphite surface predominantly as $D_{2}$ molecules which are broken down in the boundary plasma by the following reaction route [7]

$$
D_{2} \rightarrow D_{2}^{+} \rightarrow D+D^{+}
$$

Thus, for each recycled $D_{2}$ molecule, one deuterium atom is produced, which is assumed to radiate at a known photon efficiency [8].

In general, the determination of fuel and impurity influx rates from spectral intensities requires detailed knowledge of the electron temperature in the radiating region because of the $T_{e}$ dependence of the photon efficiency [5]. However, for simplicity it is assumed that the deuterium atoms and carbon ions radiate from a plasma with the same electron temperature, namely $T_{e}(a)$. This assumption is reasonable considering the short mean free paths of these species ( $\sim$ few $\mathrm{cms}$ ) in comparison with the radial scale length of the electron temperature in the boundary plasma. In the case of C II, detailed LIM carbon transport calculations (see Sec. 5) confirm this hypothesis. In the case of $D_{\alpha}$, although detailed transport calculations were not performed, the $D_{\alpha}$ photon efficiency is only weakly dependent on $T_{e}(a)[8]$ and thus the radial location of the radiating atoms is of less importance. 
The observation point for the $D_{\alpha}$ and $C \|$ emission consists of 5 lines of sight above the mid-plane at one toroidal location at the centre of bay B, Fig. 1. Global influxes are thus inferred assuming top/bottom and toroidal symmetry of the interaction with the limiter. The validity of this assumption for $D_{\alpha}$ has been verified using the CCD camera plasma viewing system. As mentioned, there is a regular periodic variation of the plasma-limiter interaction in the toroidal direction due to the shape of the limiter [9]. This is allowed for, approximately, in the determination of the absolute influxes. Considering the errors involved in using a single electron temperature in the photon efficlency conversion and geometrical eriors involved with the limiter shape, the influx determinations are estimated to be accurate only to within a factor of $\sim 2$.

The deuterium influx $\Gamma_{D}$ rises somewhat faster than linear from zero at the lowest density to $\sim 7 \times 10^{22} s^{-1}$ at the highest. The carbon influx $\Gamma_{C}$ appears to pass through a minimum at moderate density, being greater at low and high density. However, considering the factor of $\sim 2$ error mentioned above, this trend may not be significant and it would be more justified to say that the carbon influx is approximately constant within the uncertainties. At the lowest density no deuterium gas puffing is used, so that the deuterium present in the discharge originates from that stored in the graphite limiter surface before the discharge. Such low density discharges are similar to Supershot target plasmas, where much of the deuterium residing in the limitei has been purged from the system (through the vacuum pumps) by conditioning discharges [10].

At low density, the carbon influx is approximately equal to the deuterium influx. This is shown more clearly in Fig. 3 , where the effective sputtering yield from the limiter is given, i.e. $Y \equiv \Gamma_{C} / \Gamma_{D}$. The yield decreases by two orders of magnitude with increasing density from a value of $Y \sim 1$ at low density to $Y \sim 10^{-2}$ at high density. As will be discussed in detail later, the drastic change in effective yield $Y$ is a reflection of the drop in edge electron temperature which controls the energies of ions striking the limiter and thus the sputtering yields.

Figure 2 also strows the central $Z_{\text {eff }}$ and the radiated power fraction $P_{r a d} / P_{b o t}$ cluring the density scan. The $Z_{\text {eff }}$ is obtained from visible bremmstrahlung emission. It follows the effective yield $Y$, being large, $Z_{\text {eff }} \sim 4.5$, at low density and dropping to $Z_{e f f} \sim 1.3$ at high 
density. As with the yield $Y, Z_{e f f}$ is controlled primarily by the edge electron temperature through ion sputtering yields. The high yield at low density thus produces a discharge where most of the electrons are supplied by the carbon influx. The drastic reduction in yield at high density to $Y \sim 10^{-2}$ results in a relatively pure plasma with $Z_{\text {off }} 1.3$.

The radiated power fraction $P_{r a d} / P_{t o r}$ is approximately constant through the density range at a level of $\sim 0.5$, except at the highest density where MARFEs [11] develop. The abrupt increase to $P_{r a d} / P_{b o} \sim 1$ that accompanies the onset of a MARFE clearly demonstrates the thermal instability nature of MARFES. The onset of the MARFE also results in an abrupi shortening of the electron temperature e-folding distance $\lambda_{T}$ with no change in the density $\theta$-folding distance $\lambda_{n}$ as measured by the Langmuir probe.

\section{LIM Impurity Production and Transport Code}

The impurity production and transport in these discharges have been modelled with the Monte Carlo impurity code LIM [12-14]. This two-dimensional code uses as input the background plasma conditions as determined in the boundary by the probe and in the central plasma by the far-infrared interferometer $\left(n_{e}\right)$ and the ECE system $\left(T_{e}\right)$.

The code launches carbon atoms physically sputtered by incident deuterons into a plasma grid and follows the carbon ions within the plasma until they redeposit on the limiter surface. The redeposited carbon ions cause sputtering of secondary carbon atoms, with a reduced numerical weight, which are further followed. This process is continued until the yield-adjusted weight of the carbon ion is reduced to a negligible level. Both the deuteron and the carbon sputtering yields are taken from the semi-empirical sputtering yield formulae in the literature for normal incidence ions [15] (the deuteron and carbon sputtering yield enhancement factor $\eta$ is not included in this calculation, but is derived as a fitting factor as discussed below). In the plasma, parallel-field transport is assumed to be classical and governed by a pure deuteron background, while cross-field transport includes diffusion $D \sim 1 \mathrm{~m}^{2} \mathrm{~s}^{-1}$ and an inward pinch velocity $v=-2 D r / a^{2}$. The two-dimensional limiter and plasma poloidal shapes are approximated as circles. Results from the code appear in Fig. 4, appearing from the top, the effective sputtering yield on the limiter $Y \equiv \Gamma_{C} / \Gamma_{D}$ assuming no enhancement factor $\eta$, the required enhancement factor $\eta$ in the deuteron and carbon sputtering yields 
needed to fit the model with the experimental $Y$, the energy radiated per carbon atom entering the plasma $E_{r a d}$, the carbon ion particle confinement time $\tau_{p}$, and the central $Z_{\text {eff }}$ of the discharge. The experimental values for the energy radiated per carbon atom $E_{\text {rad }}$ is simply the experimental value of $P_{\text {rad }} / \Gamma_{C}$. The experimental carbon particle confinement time $\tau_{p}$ is determined using the expression $\tau_{p}=\left\langle n_{C}>V / \Gamma_{C}\right.$, where $\left\langle n_{C}>\right.$ is determined using the experimental $Z_{e f f}$ values. These are discussed in more detail in the following sections.

\section{Conversion of $C$ III Intensity to Carbon Influx Rate}

The experimentai carbon influx $\Gamma_{c}$ presented in Fig. 2 was derived using the experimental $\mathrm{C} \|$ photon intensity and converting to particle influx using the photon efficiency corresponding to the electron temperature at the LCFS, $T_{c}(a)$. Three possible problems may make such a procedure invalld. First, the photon efficiency is $T_{c}$-dependent and the $\mathrm{C} \| \mathrm{l}$ ions radiated over a range of minor radii, i.e. over a ranges of electron temperatures, thus it is not clear that the photon efficiency corresponding to $T_{e}(a)$ is a reasonable average. Second, some of the atoms sputtered from the limiter surface may not become ionized to $C$ II, but may simply deposit on the wall, thus releasing no $\mathrm{C} \| \mathrm{photons}$. Third, even if the atom is ionized in the plasma to $\mathrm{C} I 1$, it may be promptly redeposited on the limiter before it progresses to $C$ III (the theoretical photon efficiency assumes that the $C$ II loss mechanism is by ionization to $C$ III).

To ensure that the above problems are not important to the simple derivation of the carbon influx, a number of checks were performed with the LIM code. The calculated photon efficiency and dependence on $T$, of Ref. [5] were employed. The photon efficiency for $657.8 \mathrm{~nm}$ increases about a factor of $\sim 10$ for the electron temperature increasing from $10 \mathrm{eV}$ to $100 \mathrm{eV}$. The LIM code was used to confirm the validity of the useful working assumption that $T_{c}$ at the LCFS, $T_{c}(a)$, is a good approximation to the average value of $T_{c}$ in the radial zone of $C \| l$ emission $\bar{T}_{e}(C I I)$ (average in the sense that the number of influx particles per $C \|$ photon equals the photon efficiency at $\left.T_{e}=\bar{T}_{e}(C I I)\right)$. It was found that over the entire range of plasma density employed, that $T_{e}(a) \sim \bar{T}_{e}(C I I)$ to within $\pm 10 \%$, thus implying only a small error due to this effect; by con- 
trast, $\bar{T}_{e}(C I I I)(464 \mathrm{~nm})$ and $\bar{T}_{e}(C I V)(312 \mathrm{~nm})$ exceeded $T_{e}(a)$ by factors of $\sim 1.5$ and $\sim$ 2 , respectively. Thus, influx measurements based on C III or C IV intensities and the same simple assumption would seriously underestimate the influx.

In addition, the code confirmed that virtually no neutrals reached the walls before being ionized. However, about $10 \%$ of the $C^{+}$ions promptly returned to the limiter before reaching the doubly-ionized state at low density, a percentage which rose to $\sim 20 \%$ for the highest density discharges. Thus, errors are again of order $10 \%$ due to this effect. The calculated photon efficiencies themselves are, however, somewhat uncertain - sometimes by a factor of 2 or more $[5,16]$. Also, as mentioned earlier, further uncertainty is incurred because the global influx measurements assume top/bottom and toroidal symmetry. The values of $\Gamma_{D, C}$ are therefore not expected to be more accurate than to a factor of $\sim 2$.

\section{Effective Sputtering Yield}

The experimental $Y$ in Fig. 4 varies from $Y \sim 1$ at low density, where $T_{e}(a)$ is high, to $Y_{\text {eff }} \sim 10^{-2}$ at high density where the edge temperature is reduced. As discussed earlier, the edge temperature, through its effect on the incident ion energies striking the limiter, influences the sputtering yield on the limiter. The LIM code (without the sputtering yield enhancement, Fig. 4) is able to reproduce the yield at moderate to high density but fails at low density. LIM can reproduce the whole data set if a fitting factor $\eta$ is used to increase both the deuteron and carbon sputtering yields at normal incidence. $\eta$ approaches a value of 2 to 3 at low density, Fig. 4 . Under such conditions, the discharge density is maintained almost entirely by the carbon influx, which is predominantly produced by carbon self-sputtering as the self-sputtering yield $\eta Y_{C}$ approaches unity.

The cause of an enhancement of the sputtering yields at low density is not clear but could possibly be explained by an increase in the average angle of ion impact on the limiter surface which may occur when the edge ion temperature $T_{i}(a)$ becomes larger than the edge electron temperature $T_{c}(a)$. Such a temperature differential at low density has been inferred from recent JET data [17]. Another possibility, advanced by Brooks et al, is that the deuterium-depleted graphite surface at low density has a higher sputtering yield compared with deuterium-saturated graphite, a conclusion based on 
detailed modelling of the sputtering process [18]. Recent ion beam experimental results [19], infact, do not show such an enhancement due to the presence of deuterium in the carbon. With regard to the former possible explanation, it is presently difficult to pursue this theoretically, and for this reason $\eta$ has been treated as an output of the data analysis, a fitting factor, rather than as an a priori computed quantity.

Some of the difficulties in realistically modelling the sputtering process are related to our incomplete knowledge of the physics involved. For example, modelling of sputtering at the very small, glancing angles $\theta_{B}$ between the magnetic fieid and the TFTR limiter surface requires an analysis of the sheath structure for $\theta_{B}<1^{\circ}$. The existing models for finite $\theta_{B}$ cannot be extended to this regime $[20,21]$ while those which assume $\theta_{B}=0$ [22] give results which are not in quantitative agreement with experiment [23]. In addition, surface roughness has a strong influence on the laboratory (accelerator-based) measurements of yield at glancing incidence angles [24,25], introducing further uncertainty.

\section{Energy Loss due to Impurity Radiation}

The LIM-calculated energy radiated per carbon atom entering the plasma is approximately constant with plasma density at $E_{\text {rad }} \sim 2 \mathrm{keV}$, whereas the experimental value is also a.pproximately constant but higher, $E_{\text {rad }} \sim 6 \mathrm{keV}$, Fig. 4 . The discrepancy may be due to the presence of neutral hydrogen in the plasma which can significantly increase the rate of power radiated due to the effect of charge exchange. In order to test for this effect, charge exchange recombination was included in the LIM code using measured cross-sections [26] and deuterium atom density distributions estimated by DEGAS [27] for the same discharges. For the lowest density discharges $E_{\text {rad }}$ increased by $\sim 50 \%$, with negligible change at the highest density. Charge exchange recombination would therefore not appear to be capable of explaining the factor $\sim 3$ discrepancy in $E_{\text {rad }}$, however, the effect can clearly be significant and uncertainties in the cross-sections, relative collision velocities, and the neutral deuterium distributions could be affecting this conclusion. It should also be noted that the experimental values of $E_{\text {rad }}$ are based on a number of independent measurements and assumptions, e.g. concerning the emitting areas, photon efficiencies, etc., and a factor of $\sim 3$ is within the overall uncertainty limits. 


\section{Carbon Particle Confinement Time and $Z_{\text {\# }}$}

The LIM carbon particle confinement time, Fig. 4, derived by averaging the trajectories of the injected carbon ions within the plasma was found to decrease with density approximately as

$$
\tau_{p} \propto \frac{1}{\left\langle n_{e}\right\rangle}
$$

in reasonable agreement with these experimental results and others $[14,16]$. The decrease of the particle confinement time with density demonstrates the ability of a densified boundary plasma to screen impurities from the central discharge. The value of $\tau_{p}$ provides a direct indication of the impurity screening efficiency of the boundary plasma and how it varies with $\left\langle n_{e}\right\rangle$ since the cross-field diffusion coefficient $D$ and the inward pinch velocity $v$ have been kept constant (with density) in the LIM calculation. Over most of the density range the absolute disagreement between model and experiment for $\tau_{p}$ is a factor of $\sim 2$.

The LIM confinement times, along with the experimental carbon influx $\Gamma_{C}$, are used to derive the central $Z_{\text {eff }}$ in Fig. 4 using

$$
<n_{C}>=\Gamma_{C} \tau_{p} / V
$$

Again, agreement between the LIM-derived $Z_{\text {eff }}$ and the experimental values is reasonable over most of the density range; however, at the lowest density the experimental $Z_{\text {eff }}$ is significantly larger than that predicted using the derived $\tau_{p}$. This just reflects the difference between the experimental $\tau_{p}$ and the LIM predicted value. The discrepancy may be due to various factors such as an underestimation of the experimental carbon influx $\Gamma_{c}$, e.g. due to unmonitored sources, or underestimation of the inward pinch velocity. The assumption of a constant value of the cross-field diffusion coefficient, $D$, is also to be questioned, see Sec. 13.

\section{Localized Impurity Source Compared with Uniform Source}

Although the carbon impurity source in limiter discharges is typically localized, parallel transport along the magnetic field lines in the boundary can be sufficiently fast that 
as far as central contamination is concerned, the impurity source is as if it were poloidally and toroidally uniform. Under this condition it can be shown $[14,28,29]$ that the particle confinement time is

$$
\begin{aligned}
\tau_{p}^{U N} & =f\left(\lambda_{i z}+\lambda_{\text {SoL }}\right] a / 2 D & & \lambda_{i z}>0 \\
& =f \lambda_{s o L} \exp \left(\lambda_{i z} \lambda_{\text {SoL }}\right) a / 2 D & & \lambda_{i z}<0
\end{aligned}
$$

where $\lambda_{i 2}$ is the ionization depth inside the LCFS (which can be negative at high density since the majority of ionization occurs within the $S O L$ ) and where $\lambda_{s o L}$ is the impurity density scrape-off length given by

$$
\lambda_{\text {soL }}=\left[D \tau_{\text {soL }}\right]^{1 / 2}
$$

where $\tau_{s o L}$ is the dwell time of the impurity ions in the SOL. In this section we explore the degree to which the TFTR bumper limiter can be thought of as a uniform source as far as central contamination is concerned.

LIM has been used to determine $\bar{\lambda}_{i s}$ by averaging the radial location of neutral ionization. $\lambda_{S O L}$ has been calculated using LIM values for the average /well time in the SOL $\bar{\tau}_{S O L}$. Both of these have been plotted in Fig. 5 as functions of density $\left\langle n_{e}\right\rangle . \bar{\lambda}_{i z}$ decreases with density, as expected, and becomes negative as most of the ionization occurs in the SOL, whereas the impurity density e-folding distance $\lambda_{S O L}$ decreases only slightly with density. Also shown in Fig. 5 are particle confinement times as determined by LIM and using Eqn. 3 substituting the LIM values for $\bar{\lambda}_{i z}$ and $\lambda_{\text {soL }}$. The LIM-calculated values of particle confinement time $\tau_{p}$ allow, of course, for the actual poloidal non-uniformities in influx and outflow and so a comparison with $\tau_{p}$ calculated assuming uniformity, Eqn. 3, i.e. $\tau_{p}^{U N}$, provides an indication of the degree of localization of the impurity influx/loss process. Results, given in Fig. 5 , show that at low $\left\langle n_{e}>\right.$ the impurity transport is approaching spatial uniformity. Although $\bar{\lambda}_{\text {is }}$ for low density is only $\sim 1 \mathrm{~cm}$, cross-field diffusion is sufficiently slow that the ions can travel along field lines a considerable distance before their radial transport brings them into effective contact with the sink action of the SOL. At high density, on the other hand, it is clear that the impurity 
transport is quite localized: $\tau_{p}^{U N}$ is a poor approximation to $\tau_{p}$ and $\bar{\lambda}_{i z}$ is $-1 \mathrm{~cm}$ to $-2 \mathrm{~cm}$. Under these conditions, the typical impurity trajectory is confined to the region near the limiter.

\section{Sink Action of SOL Plasma}

The LIM code was used to further investigate the nature of the impurity ion removal mechanisms and their effect on the particle confinement time (i.e. the central carbon concentration) by (in the code) turning off,

(A) the deuterium flow $v_{S O L}$ and electrostatic field $E_{S O L}$ along the magnetic field in the SOL

(B) parallel diffusion $D_{p a r}$ along the magnetic field in the $\mathrm{SOL}$

For the discharge examined the density was $\left\langle n_{e}\right\rangle=1.5 \times 10^{19} \mathrm{~m}^{-3}$. The LIM-calculated values of $\tau_{p}$ and $\bar{\tau}_{s O L}$ are given in Table 2. Turning off the deuterium flow and the electrostatic field in the SOL removes any net force which would sweep the impurity ions along the magnetic field in the SOL to the limiter sink and thus has a large effect on $\bar{\tau}_{\text {soL }}$ (factor $\sim 5$ ), but a weaker effect on the overall particle confinement time $\tau_{p}$ (factor $\sim 2$ ). The ions can still reach the limiter, but only via parallel-field transport simply due to parallel diffusion; however, as can be seen from Table 2, turning off parallel diffusion likewise has only a weak effect on $\tau_{p}$, whereas turning off both parallel diffusion and the net forces only causes $\tau_{p}$ to increase by $\sim 50 \%$. The reason that a more dramatic increase in particle confinement (or central impurity levels) was not found, despite completely removing the parallel-field sink action of the SOL, is that cross-field motion is extremely important for a very large limiter such as the TFTR bumper limiter. Even for a low density discharge, where the impurity ions can disperse along the magnetic field inside the LCFS quite appreciably, the limiter lies only a few centimetres outside the LCFS over a large fraction of the entire poloidal extent of the plasma and is thus an important cross-field sink. This can be demonstrated using the following simple calculation; the value of $\bar{\tau}_{S O L}$ for the case of no parallel-field sink action was $1.1 \mathrm{~ms}$; for $D=1 \mathrm{~m}^{2} / \mathrm{s}$ the radial spread in this time is $\Delta r \sim\left[2 D \bar{\tau}_{s o L}\right]^{1 / 2} \sim 5 \mathrm{~cm}$, which explains the strong cross-field sink action for the TFTR bumper limiter since the limiter-L.CFS separation is only of this order over most of the limiter surface. 


\section{Comparison with Rail Limiter}

The results of the last section are considerably different than what would be expected for a toroidal rail limiter (i.e. a flat plate, see Fig. 6) located at the inside midplane of TFTR, assuming the same plasma conditions (the plane of such a limiter would be in the mid-plane of the tokamak, extending radially out from the inner wall by, an assumed, $20 \mathrm{~cm}$ ). Results for this case are also shown in Table 2. As can be seen, turning off the parallel sink action would now cause $\tau_{p}$ to increase by a factor of $\sim 17$ ! The value of $\bar{\tau}_{S O L} \sim 18 \mathrm{~ms}$ gives a radial spread $\Delta r \sim\left[2 D \bar{\tau}_{s o l}\right]^{1 / 2} \sim 19 \mathrm{~cm}$, i.e. approximately the distance from the LCFS to the wall in this hypothetical case.

Comparison of the two limiter geometries in Table 2 highlights an important difference between limiters whose surface normal is nearly radially inward, e.g. the TFTR bumper limiter, and a limiter whose surface normal is directed poloidally (the toroidal rail case of Table 2) or toroidally (e.g. a flat plate poloidal fing limiter). Comparing the first case in Table 2, one sees that the TFTR bumper limiter is $\sim 2$ times more effective at contaminating the core plasma than a toroidal rail limiter would be. The reason is simply that an inward-facing limiter directs sputtered particles radially into the plasma, while side-facing limiters do not. (This aspect of limiter shape has been further examined experimentally and theoretically in the Impurity Control Limiter (ICL) study on DITE [30].) The inward-facing limiter, however, provides a larger area for heat removal than does a side-facing limiter and thus the trade-off regarding limiter shape, is clearly one between power handling and impurity control. In TFTR during high power operation heat removal is of critical importance and thus the limiter design has been optimized for heat removal.

\section{SOL Forces}

With regard to the nature of the parallel sink action of the SOL, unfortunately, there are few measurements [31] of the two dominant forces in limiter tokamaks. These are friction (i.e. that associated with the plasma flow velocity along the magnetic field in the SOL towards the limiter $v_{\text {SoL }}$ ) and the electrostatic electric field $E_{\text {Sol }}$; temperature gradient forces are not thought to usually be important in limiter tokamaks [14]. Models are available which predict the magnitude and spatial variation of $v_{S O L}$ and $E_{S O L}$ but in the absence of experimental confirmation their reliability cannot be assessed. The LIM 
results presented here assimmed that $y_{S O L}$ increased linearly from zero at the stagnation point (i.e. at the outside mid-plane on TFTR) to the acoustic speed $c_{s}$ at the limiter, while $E_{S O L}$ increased linearly from zero to $E_{S O L}=-k T_{c} / 2 L$, where $L=\pi R_{0} q_{\psi}$ is the connection length. For a low density discharge, the effect on $\tau_{p}$ of changing the assumptions about $v_{S O L}$ and $E_{S O L}$ were examined. When a simple fluid model, predicting non-linear variations of $v_{S O L}$ and $E_{S O L}$ [13] was employed instead of the simple prescription above, $\tau_{p}$ was found to increase by only $10 \%$. When it was assumed that $v_{S O L}=c_{S}$ and $E_{S O L}=-k T_{e} / 2 L$ throughout the entire SOL, $\tau_{p}$ decreased by only $2 \%$. The reason for the small changes is partially, as already noted, the cross-field sink action, which is quite important in TFTR. As can be seen from Table 2, however, the parallel sink action is about equally important as the cross-field sink and so the explanation is more general, and less specific to the TFTR limiter shape. The impurity ions are mainly influenced by the value of $v_{S O L}$ and $E_{S O L}$ near the limiter, even for a low density case where the dispersal of the impurity ions away from the limiter is greatest. Virtually all models predict that $v_{s o L} \sim c_{S}$ at the limiter, hence the small differences in $\tau_{\rho}$ caused by the different models (also, generally the electrostatic force on impurity ions is found to be small compared with the friction force).

\section{Spatially-Varying Transport}

On other tokamaks [16] various experiments have indicated that cross-field transport favours the low-field side, i.e. $R>R_{0}$. The confinement time $\tau_{p}$ is approximately inversely proportional to the cross-field diffusion coefficient $D$ but the sensitivity is almost solely to the value of $D$ in the region where the carbon neutrals are ionized. For TFTR, this is on the high-field side $\left(R<R_{0}\right)$, and somewhat inside of the LCFS, particularly for low density discharges. It was suspected that spatial variations in the crossfield diffusion coefficient may explain the discrepancy, pronounced at the lowest density, between the LIM-calculated and experimental values of $\tau_{p}$ and $Z_{e f f}$, Fig. 4 . To test this the LIM code was run with spatially varying values of the cross-field diffusion coefficient $D$. For a low density discharge with $\left\langle n_{\mathrm{e}}\right\rangle=1.1 \times 10^{19} \mathrm{~m}^{-3}$, the plasma was divided into four regions with the following assumed values of the cross-field diffusion coefficient $D$,

(I) inside the LCFS $r<a$, low-field side, $R>R_{0}, D=1 \mathrm{~m}^{2} \mathrm{~s}^{-1}$ 
(II) Inside the LCFS $r<a$, high-field side, $R<R_{0}, D=0.3 m^{2} s^{-1}$

(III) in the SOL $r>a$, low-field side, $R>R_{0}, D=3 m^{2} s^{-1}$

(IV) in the SOL $r>a$, high-field side, $R<R_{0}, D=0.3 \mathrm{~m}^{2} \mathrm{~s}^{-1}$

This change causes the LIM-calculated $\tau_{p}$ to increase from $21 \mathrm{~ms}$, the value for $D=1 \mathrm{~m}^{2} \mathrm{~s}^{-1}$ everywhere, to $71 \mathrm{~ms}$, essentially equal to the experimental value in Fig. 4.

The value assumed for the cross-field diffusion coefficient $D$ in the SOL did not affect $\tau_{p}$ significantly; also the assumption of $D=3 \mathrm{~m}^{2} \mathrm{~s}^{-1}$ throughout the SOL gave about the same value of $\tau_{p}$ as for the assumed poloidal-variation, above. The reason for considering such large values of diffusion coefficient in the SOL, however, was to demonstrate that large $\theta$-folding distances $\lambda_{n}$, Fig. 2 , which imply large cross-field diffusion coefficients, can be consistent with low $D$ values inside of the LCFS $(r<a)$ on the high-field side $\left(R<R_{0}\right)$, thus with large values of $\tau_{p}$ and $Z_{\text {aff }}$.

It may be noted from Fig. 5 that the calculated values of the impurity e-folding length $\lambda_{\text {soL }}$ are rather shorter than the plasma $\theta$-folding lengths $\lambda_{n}$ in Fig. 2 . Unfortunately, no independent measurements of $\lambda_{S O L}$ are available, but one might expect $\lambda_{\text {soL }} \sim \lambda_{n}$. The $\lambda_{\text {soL }}$ values in Fig. 5 , however, were for $D \sim 1 \mathrm{~m}^{2} \mathrm{~s}^{-1}$ everywhere and the calculated impurity scrape-off lengths can be increased, without significant change to the calculated $Z_{\text {eff }}$ and $\tau_{p}$ values, by assuming large values of $D$ in the SOL logether with small $D$ values in the confined plasma inboard of the limiter. This effect was demonstrated by changing the SOL diffusion coefficient from $D=1 \mathrm{~m}^{2} \mathrm{~s}^{-1}$ to $D=10 \mathrm{~m}^{2} \mathrm{~s}^{-1}$ for a discharge with $\left\langle n_{e}\right\rangle=1.5 \times 10^{19} \mathrm{~m}^{-3}$, keeping $D=1 \mathrm{~m}^{2} \mathrm{~s}^{-1}$ inboard of the LCFS. The increase in the scrape-off layer diffusion coefficient actually caused a small increase in the particle confinement time $\tau_{p}$, by $\sim 50 \%$, due to the $\lambda_{\text {soL }}$ term in the numerator of Eqn. 3. The value of $\lambda_{\text {soL }}$ was increased substantially, from $13 \mathrm{~mm}$ to $40 \mathrm{~mm}$, approximately equal to the $\lambda_{n}$ values of Fig. 2 .

While other interpretations of the data cannot be ruled out, the present observation of high particle confinement times $\tau_{p}$ together with large $\theta$-folding distances $\lambda_{n}$ at low density, can be taken as further evidence of spatially varying cross-field transport. As indicated, transport favours the low-field side poloidally, possibly due to a ballooning 
effect [16]. With regard to the radial variation of the diffusion coefficient, studies in the confined plasma on TFTR [32,33], i.e. Inboard of the :-CFS, have shown that transport (heat, momentum, particle diffusivities) increases strongly toward the edge. The spatial variations found here are thus in accord with other studies.

\section{Direct Comparison of LIM with the Simple Model}

Also shown in Fig. 4 is the comparison where possible between a simple analytic model [34] with the LIM results; specifically, the effective sputtering yield $Y$ assuming no enhancement, the sputtering yleld enhancement factor $\eta$ and the $Z_{\text {eff. }}$. The analytically derived $Y$ and $\eta$ are determined using the following expression [34]

$$
Y=\frac{\Gamma_{C}}{\Gamma_{D}}=\frac{\eta Y_{D}}{1-\eta Y_{C}}
$$

where $Y_{D}$ and $Y_{C}$ are the sputtering yields for deuterons and carbon ions at normal incidence. The deuterons are assumed to have an impact energy of $5 T_{e}$ while the carbon ions are assumed have a charge state of 4 and an energy of $14 T_{e}$. The analytic $Z_{\text {eff }}$ is determined using the experimental effective sputtering yield $Y$ and the expression [34]

$$
\frac{\left\langle n_{C}\right\rangle}{\left\langle n_{D}\right\rangle}=Y \frac{\lambda_{C}}{\lambda_{D}}
$$

where $\lambda_{C} / \lambda_{D}$ is the ratio of effective penetration distances of the carbon and the deuterlum and is assumed here to be constant, ie $\lambda_{C} / \lambda_{D} \sim 1 / 2$ [34]. In the cases of the effective sputtering yield $Y$ and the enhancement factor $\eta$, good agreement between the analytic values and the LIM values is obtained. In the case of the $Z_{\text {eff }}$, even better agreement with experiment is obtained with the analytic result than with the LIM result. This is primarily due to the fact that $Z_{\text {eff }}$ is a relative measure of contamination whereas the LIM calculation attempts to determine the absolute impurity content of the discharge which is governed by many factors, including the possibility of poloidal variations in the diffusion coefficient. The analytic calculation, on the other hand, avoids this by considering only the relative influx $\Gamma_{C} / \Gamma_{D}$ taken from experiment and a relative effective penetration ratio, i.e. $\lambda_{C} / \lambda_{D} \sim 1 / 2$. 


\section{Summary}

To summarize the main experimental and modelling results of this study:

(1) The experimental results are consistent with physical sputtering by deuteron and carbon lons being the dominant impurity release mechanism, provided an enhancement in the normal incidence yields is allowed for, $\eta \sim 2$. This enhancement may be due to the edge ion temperature being greater than the edge electron temperature [17].

(2) At low density the carbon self-sputtering yield $\eta Y_{C}$ approaches unity and thus the discharge density is maintained primarily by the carbon influx.

(3) The energy radiated per carbon atom $E_{\text {rad }}$ is constant with density but higher than that predicted using the LIM code. A small, but significant fraction of the factor of 3 discrepancy can be related to the presence of atomic deuterium which is known to enhance the impurity radiation rate through charge-exchange with the impurity ions.

(4) The carbon particle confinement time $\tau_{p}$ decreases with density as $\tau_{p} \propto<n_{e}>^{-1}$, demonstrating the increased screening by the plasma boundary as density increases.

(5) The central $Z_{\text {eff }}$ is controlled to a large degree by the effective sputtering yield at the limiter $Y \equiv \Gamma_{C} / \Gamma_{D}$, which in turn is influenced by the edge temperature $T_{c}(a)$ and the sputtering yield enhancement $\eta$.

(6) At low density, significant quantities of impurities produced at the limiter can spread along the magnetic field sufficient distance that the effective source for the central plasma is as if it were poloidally uniform. At higher density, impurity ion transport is quite localized, with the typical ion trajectory being confined close to the limiter.

(7) Over most of the density range, the limiter sink action for impurities is primarily due to cross-field diffusion directly onto the limiter surface. Only at the lowest 
density, where parallel dispersal is fast, is the parallel field sink action significant. This behaviour is significantly different than would occur for a rail limiter, for example, where the parallel sink action would be dominant. The TFTR limiter geometry, because it is optimized to reduce surface power density during high power operation, tends to be more effective at contaminating the central plasma than a rall limiter.

(8) The observation at low density of high particle confinement times $\tau_{p}$ despite long SOL e-folding distances at the outside mid-plane can be explained by a spatial variation of the cross-field diffusion coefficient; small on the high-field side of the plasma and large on the low-field side and radial variation, larger in the SOL, smaller in the confined plasma.

\section{References}

(1) A T Ramsey and S L Turner, Rev Sci Instrum 58 (1987) 1211.

(2) S S Medley, D L Dimock, S Hayes et al, Rev Sci Instrum 56 (1985) 1873.

(3) KW Hill and the TFTR Team, 11th Int Conf on Plas Phys and Contr Fusion, IAEA Kyoto, 1986, IAEA-CN-47/A-IV-2, Vol I, 207.

(4) M Ulrickson, J Nucl Mat 176/177 (1990) 44.

(5) K Behringer, H P Summers, B Denne, M Forrest and M Stamp, Plas Phys Contr Fus 31 (1989) 2059.

(6) S J Kilpatrick, C S Pitcher, H F Dylla, et al, J Vac Sci Techn A8 (1990) 1767.

(7) M F A Harrison, in "Physics of Plasma-Wall Interactions in Controlled Fusion", Eds. D E Post and R Behrisch, NATO ASI Series, Plenum Press, New York (1986).

(8) L C Johnson and E Hinnov, J Quant Spectrosc Radiat Transfer 13 (1973) 333.

(9) RT McGrath and J N Brooks, J Nucl Mat 162-164 (1989) 350.

(10) H F Dylla, P H LaMarche, M Ulrickson et al, Nucl Fusion 27 (1987) 1221.

(11) B Lipschultz, J Nucl Mat 145-147 (1987) 15. 
(12) P C Stangeby and C Farrell, Plas Phys and Contr Fusion 32 (1990) 677.

(13) P C Stangeby, C Farrel, S Hoskins and L Wood, Nucl Fusion 28 (1988) 1945.

(14) P C Stangeby, J Nucl Mat 176/177 (1990) 51.

(15) J Bohdansky, Sputtering, in "Data Compendium for Plasma-Surface interactions", Nucl Fusion Speclal Issue 1984, 61.

(16) P C Stangeby and G M McCracken, Nucl Fusion 30 (1990) 1225.

(17) S K Erents, S Clement, P J Harbour et al, J Nucl Mat 176/177 (1990) 301.

(18) J N Brooks, D K Brice, A B DeWald and R T McGrath, J Nucl Mat 162-164 (1989) 363.

(19) E Pasch, P Bogen and Ph Mertens, J Nud Mat 176/177 (1990) 455.

(20) R Chodura, Phys Fluids 25 (1982) 1628.

(21) A B DeWald, A W Bailey and J N Brooks, Phys Fluids 30 (1987) 267.

(22) K Theilhaber and C K Birdsall, Phys Fluids B1 (1989) 2244.

(23) G F Matthews, S J Fielding, G M McCracken et al, Plas Phys and Contr Fusion 32 (1990) 1301.

(24) J Roth, in "Physics of Plasma-Wall Interactions in Controlled Fusion", Eds. DE Post and R Behrisch, NATO ASI Series, Plenum Press, New York (1986).

(25) A A Haasz, J W Davis and C H Wu, J Nucl Mat 162-164 (1989) 915.

(26) P T Greenlarid, AERE-report 11281 (1984), Harwell, Oxfordshire, U.K., "Low Energy Charge Capture Cross Sections".

(27) D B Heifetz, in "Physics of Plasma-Wall Interactions in Controlled Fusion", Eds. D E Post and R Behrisch, NATO ASI Series, Plenum Press, New York (1986).

(28) W Engelhardt and W Feneberg, J Nucl Mat 76/77 (1978) 518.

(29) DE Post and K Lackner, in "Physics of Plasma-Wall Interactions in Controlled Fusion", Eds. D E Post and R Behrisch, NATO ASI Series, Plenum Press, New York (1986).

(30) G F Mathews, S J Fielding, G M McCracken, et al, Nucl Fusion 28 (1988) 2209. 
(31) C S Pitcher, P C Stangeby, D H J Goodall et al, J Nucl Mat 162-164 (1989) 337.

(32) S D Scott, V Arunasalam, C W Barnes et al, Phys. Fluids B2 (1990) 1300.

(33) E J Synakowski, B C Stratton, P C Etthimion et al, Phys Rev Letters 65 (1990) 2255.

(34) C S Pitcher, P C Stangeby, R V Budny et al, to be published in Nucl Fusion (1992). 


\section{Table and Figure Captions}

Table 1 An investigation of the sink action of the scrape-off layer as determined by LIM for discharge 45426 with $\left\langle n_{e}\right\rangle=1.5 \times 10^{19} \mathrm{~m}^{-3}$, assuming a cross-field diffuslon coefflcient of $D \sim 1 \mathrm{~m}^{2} \mathrm{~s}^{-1}$ and an Inward pinch velocity of $v=-2 D \mathrm{r} / \mathrm{a}^{2}$. The effect of the $S O L$ net forces $\left(v_{S O L}, E_{S O L}\right)$ and parallel diffusion $\left(D_{p a r}\right)$ are shown as they Influence the carbon particle confinement time $\tau_{p}$ and the average carbon lon dwell time in the SOL $\bar{\tau}_{\text {soL }}$.

Fig. 1 Schematlc cross-sectlon dlagram of the TFTR vessel showing the arrangement of the toroldal graphite bumper limiter, the reciprocating Langmulr probe and the pololdal $D_{\alpha}$ C II telescope array.

Fig. 2 Experimental boundary plasma conditions from the Ohmic density scan sequence of discharges as a function of volume-averaged density $\left\langle n_{e}\right\rangle$ : the

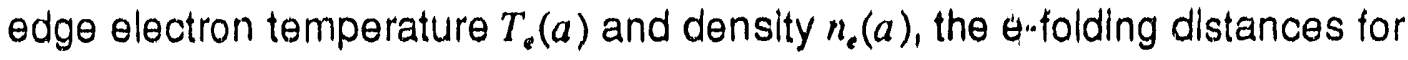
electron temperature $\lambda_{T}$ and density $\lambda_{n}$ in the $S O L$ at the position of the probe, the deuterlum $\Gamma_{D}$ and carbon $\Gamma_{C}$ particle influxes from the limiter, the central $Z_{\text {eff }}$ and the radlated power fraction $P_{\text {rad }} / P_{\text {tor }}$. The lines drawn through the data are simply to aid the eye (l.e. not model predic'lons).

Fig. 4 A comparison between the experimental bounidary conditions and predictions from the LIM code as a function of volume-ave aged density $\left\langle n_{e}\right\rangle$ : effective sputtering yield $Y \equiv \Gamma_{C} / \Gamma_{D}$ assuming no enhancr.eent in the sputtering yield, the sputtering yield enhancement factor $\eta$ required to fit the predicted yield with experiment, the energy radiated per carbon atom influx $E_{r a d}$ the carbon particle confinement time $\tau_{p}$ and the the central $Z_{e f f}$. Also shown for comparison with the LIM results are the corresponding analytic values from the simple model for $Y \equiv \Gamma_{C} / \Gamma_{D}, \eta$ and $Z_{\text {eff }}$. 
Fig. 5 LIM results as a function of volume-averaged density $\left\langle n_{e}\right\rangle$ : the average radial location with respect to the LCFS (i.e. positive means inboard of the LCFS and negative means in the SOL) of neutral ionization $\bar{\lambda}_{i z}$, the impurity scrape of length as determined using Eqn. 4 and the LIM-calculated value for the average dwell time in the SOL $\bar{\tau}_{S o L}$, the carbon particle confinement time calculated with Eqn. 3 assuming a uniform influx $\tau_{p}^{\nu N}$, the carbon particle confinement time determined by LIM taking into account the poloidal geometry $\tau_{p}$. The lines drawn through the data are simply to ald the eye.

Fig. 6 Schematlo diagrams for comparison of the existing TFTR bumper limiter with a toroidal rall limiter. 
Table 1

SOL Assumptions

$v_{S O L}, E_{S O L}$

on

off

on

off
$D_{p a r}$

on

on

off

off
TFTR Bumper Limiter

$\bar{\tau}_{\text {SOL }}$

(ms)

18

35

15

28 (ms)

0.19

2.0

0.068

1.1
Ioroldal Rail Limiter

$\tau_{p}$

$\bar{\tau}_{\text {SOL }}$

(ms)

(ms)

8.4

0.19

39

4.0

5.3

0.10

18 

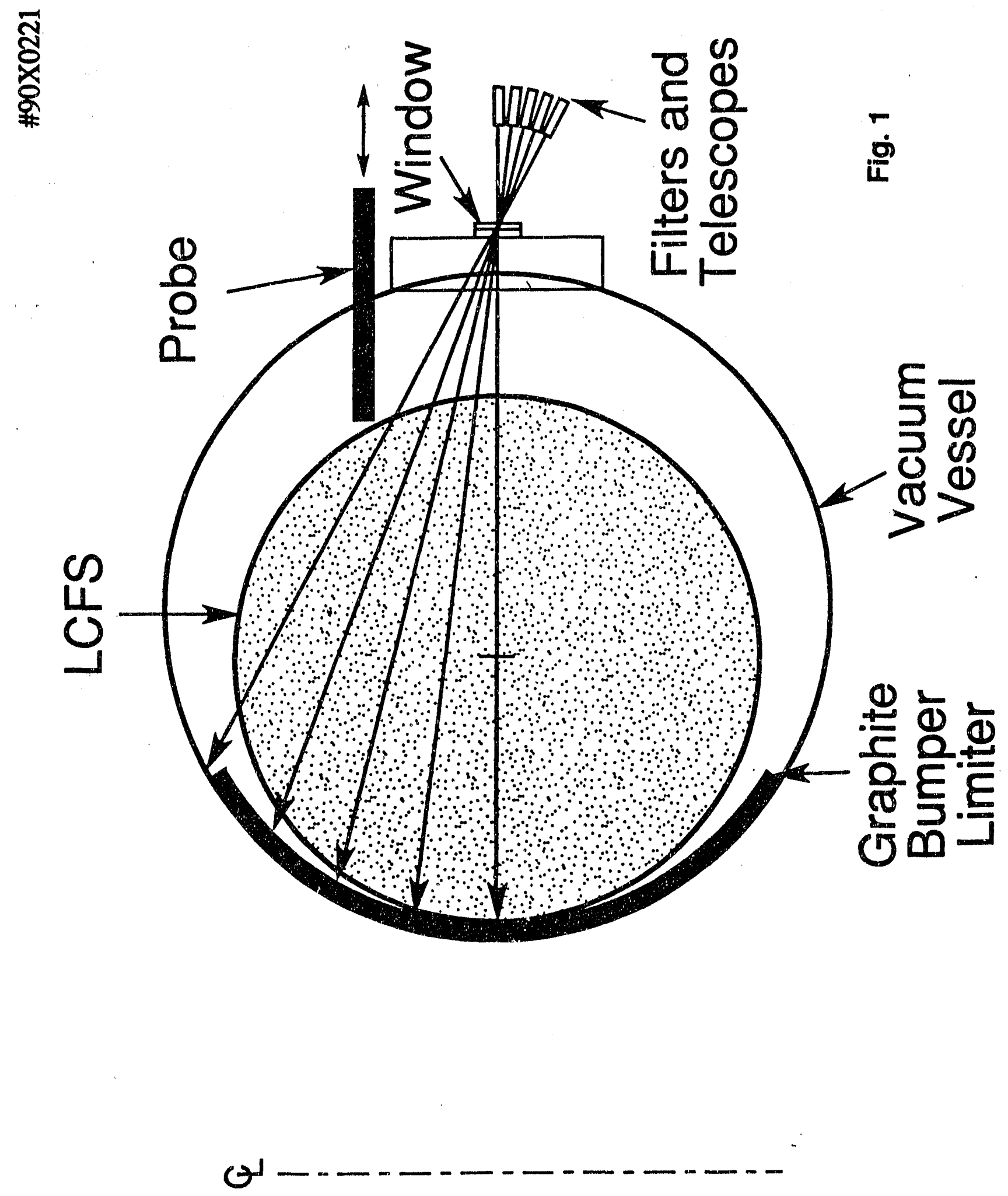


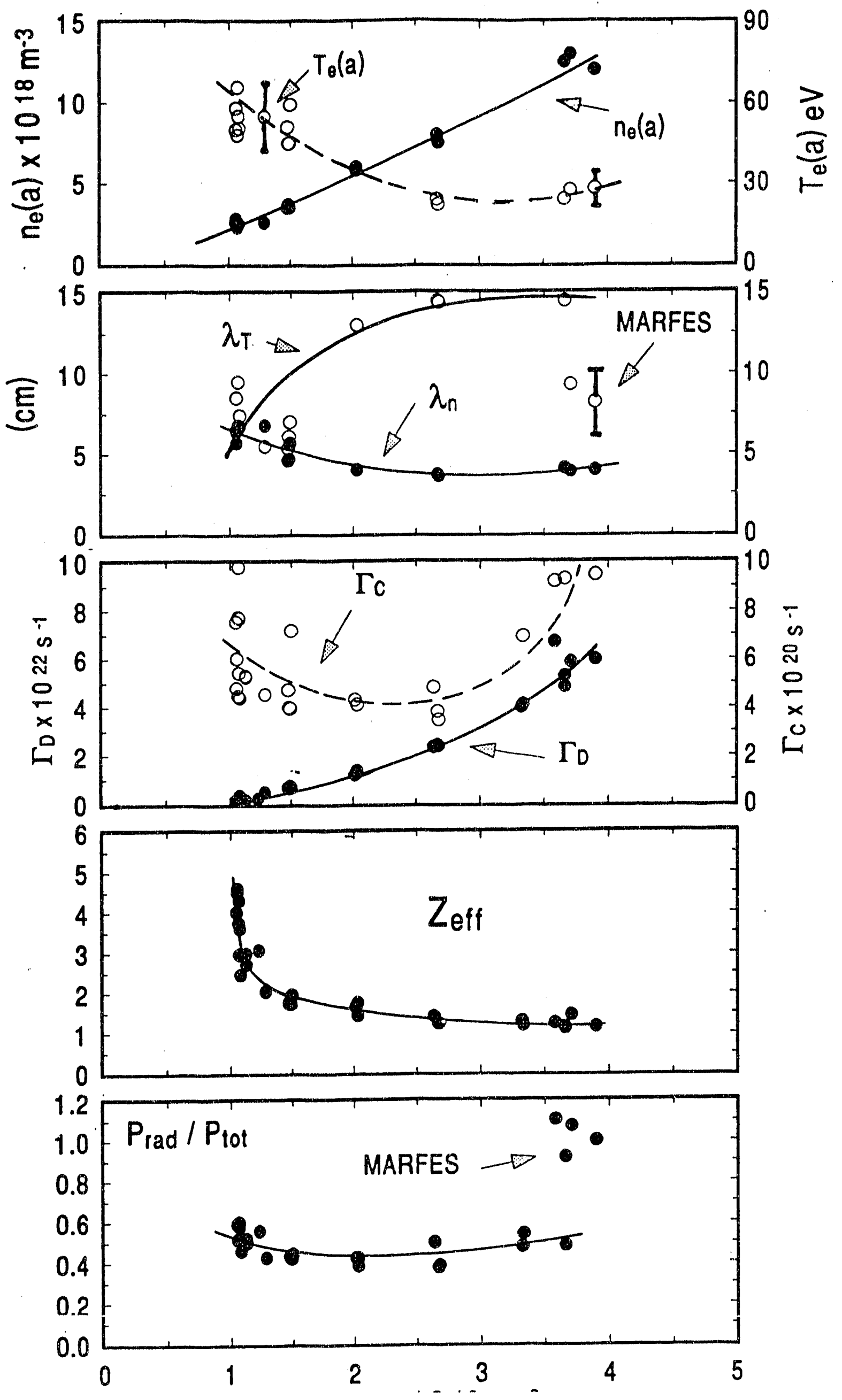

Fig. 2 


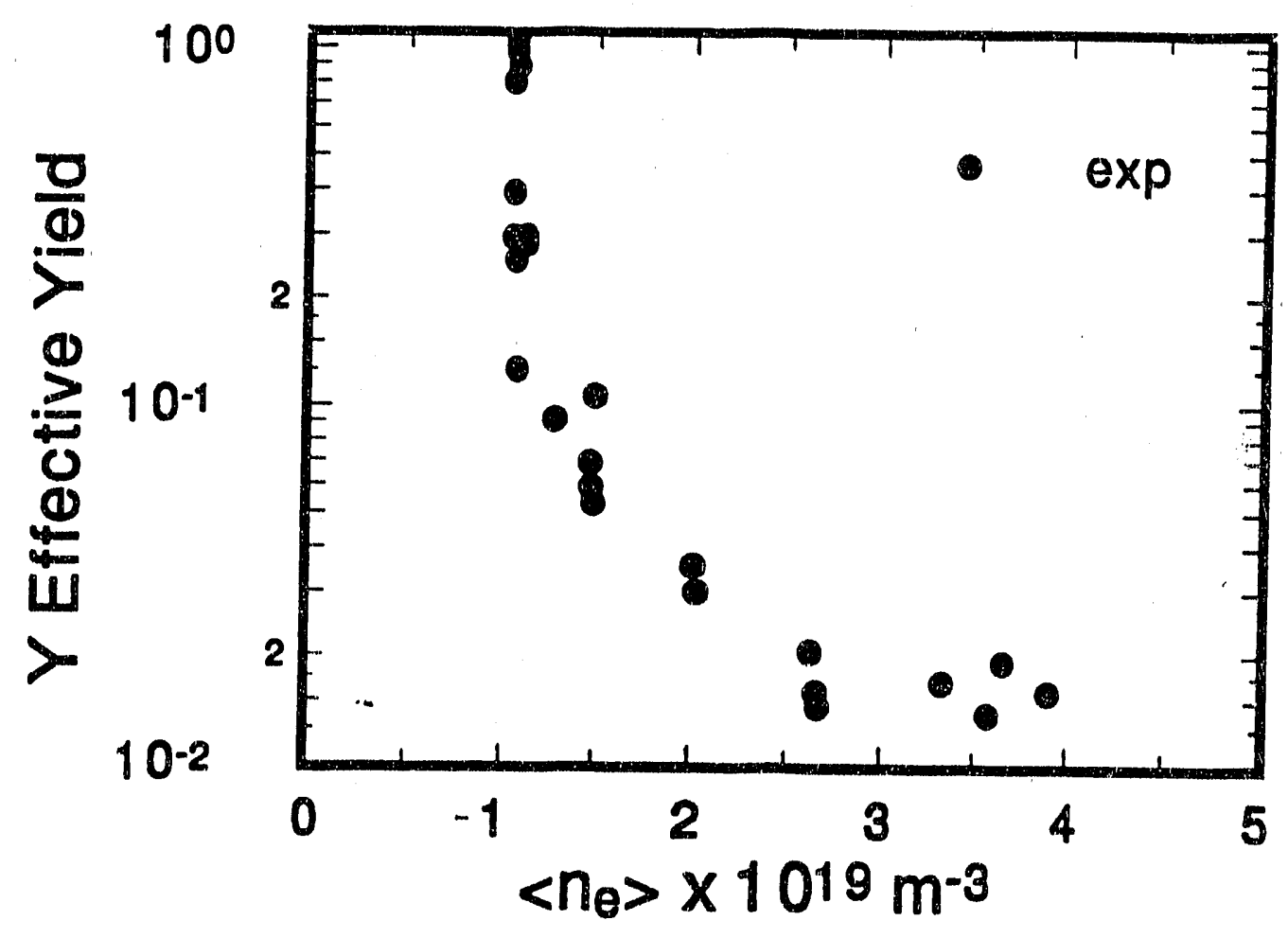

Fig. 3 


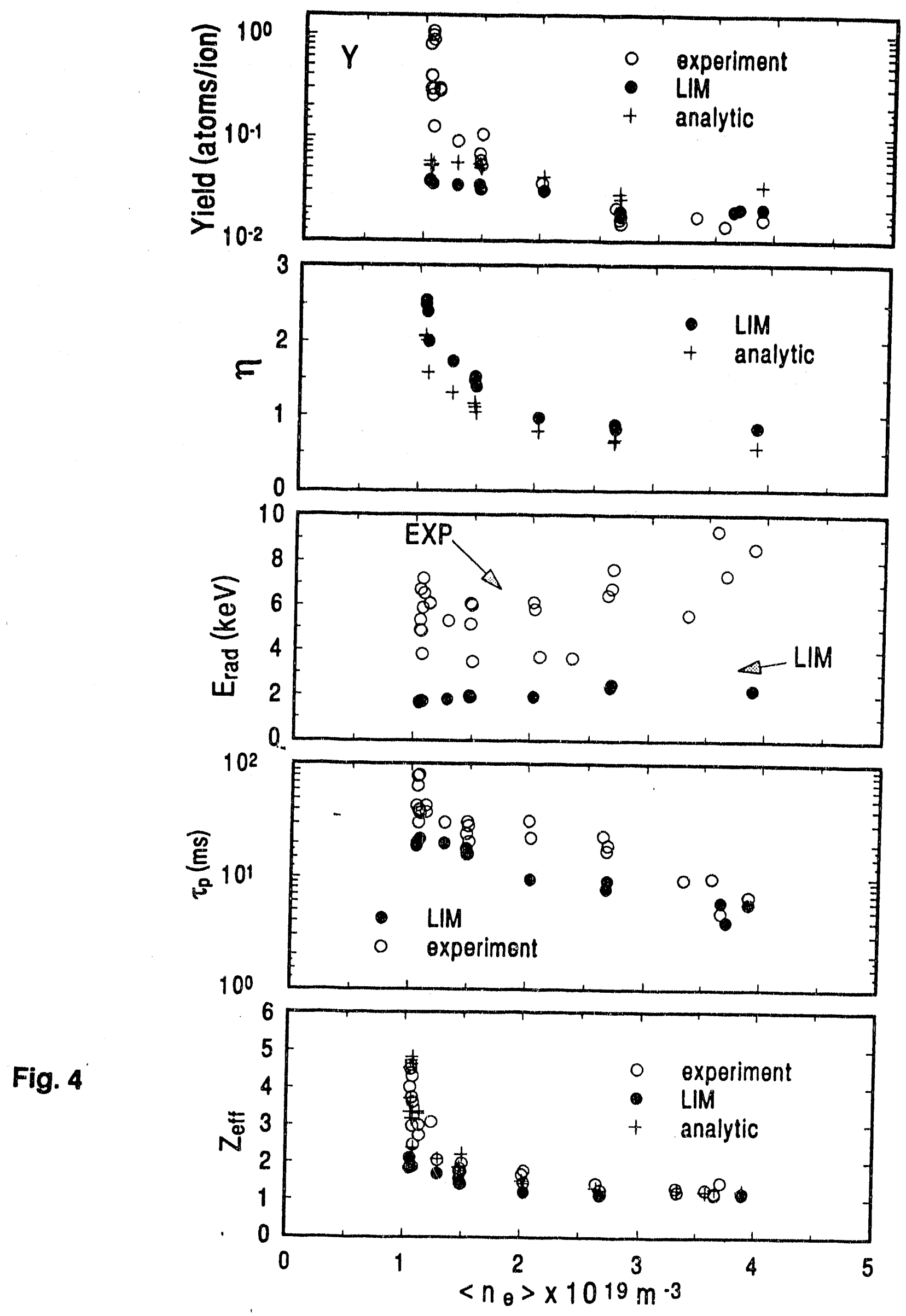



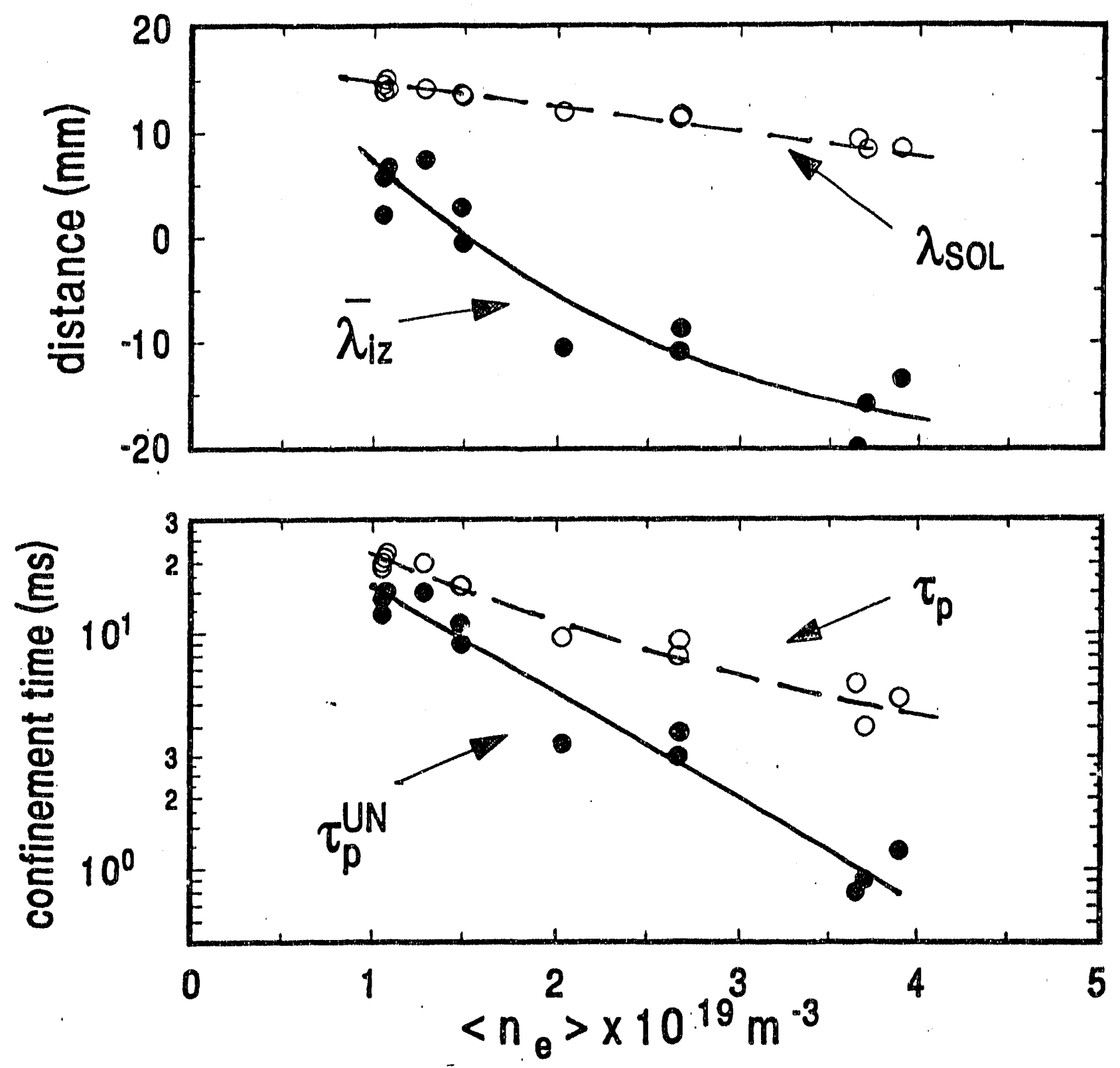

Fig. 5 


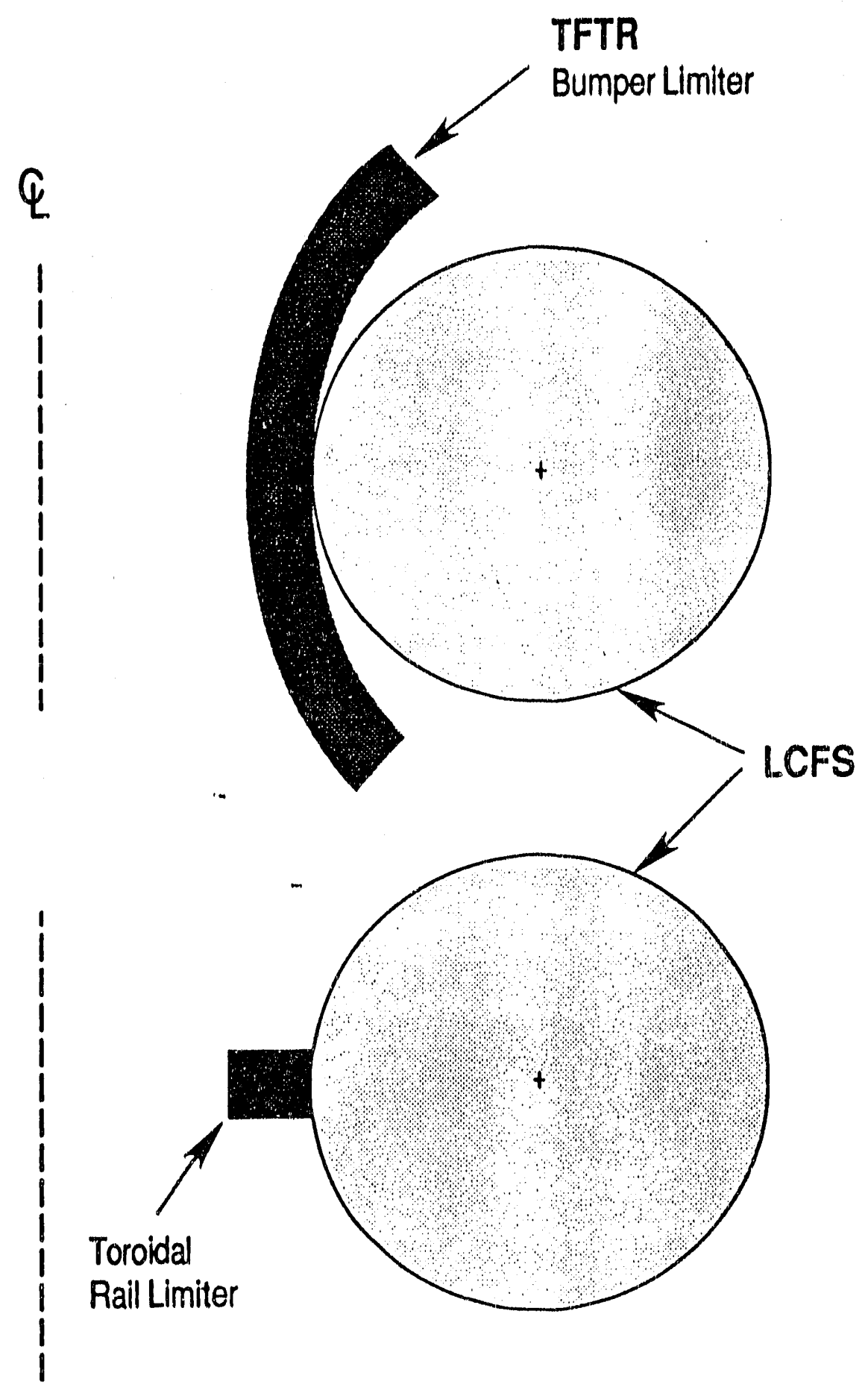

Fig. 6 


\section{(II) Particle and Power Fluxes to Surfaces for a Glanc- ing Magnetic Field}

C S Pltcher", P C Stangeby and J D Elder

University of Toronto, Canada

* Canadian Fusion Fuels Technology Project

1 Introduction

It is usually assumed in the design of fusion devices that the particle $\Gamma$ and power $P$ flux densities incident on limiters or divertor plates are proportional to the product of the parallel field flux density and the projected area perpendicular to the field lines, that is,

$$
\begin{aligned}
& \Gamma_{\text {SURFACE }} \propto \Gamma_{p a r} \cos \theta \\
& P_{\text {SURFACE }} \propto P_{p a r} \cos \theta
\end{aligned}
$$

where $\theta$ is the angle between the surface normal and the magnetic field and "par" indicates parallel fleld fluxes. These relationships may be called the "Cosine Law".

The Cosine Law has been assumed in the design of limiters and divertor plates for many years and has leard to the use of glancing angles between the surface and the magnetic field to reduce power densities on critical first-wall components, thus $70^{\circ}<\theta<90^{\circ}$. In contemporary large, high-powered machines the parallel power densities flowing in the SOL can be very large, $>100 \mathrm{MW} \mathrm{m}^{-2}$, and thus angles must be made very glancing to reduce the surface power density to an acceptable level, $|\theta| 90-\theta \mid<5^{\circ}$. ITER takes this approach to an extreme with $|90-\theta| \sim 1^{\circ}$.

In a recent study on DITE [1], it was shown that the Cosine Law was not obeyed for angles $|90-\theta|<3^{\circ}$. In another study on DIII [2], in apparent contradiction, it was found that the power flux density did follow the Cosine Law down to very small angles, $|\theta| 90-\theta \mid \sim 0.5^{\circ}$. In this section we present new results on the particle flux to the TFTR bumper limiter as deduced from $D_{\alpha}, \mathrm{He} I$ and $\mathrm{C} \|$ emissions measured with the poloidal 
array and with a CCD camera. The results again show that over most of the limiter surface, where $|90-\theta|<3^{\circ}$, the Cosine Law is not obeyed. In fact, the results indicate no angular dependence within this range.

\section{Experiment}

The viewing geometry for the array (Bay B) is shown in Fig. 1 of Section I. The camera (Bay P) vlewing geometry is shown in Fig. 1 of the present section.

As mentioned in Section I, the toroidal limiter has a periodic structure in the toroidal direction which repeats at each bay. There are 20 such bays equi-spaced around the torus, corresponding to the 20 toroidal field coils. Fig. 2 approximately shows the field of view (In the absence of plasma) of the camera, looking from the outside midplane towards the inner bumper limiter along a major radius. Shown on the sketch is the co-ordinate system used in this section; " $z$ " igfers to the vertical distance above the midplane, while " $y$ " refers to the horizontal distance from the middle of Bay $P$.

In Fig. 3 we compare the toroidal shape of the limiter at the midplane with the last close magnetic flux surface (LCFS) to demonstrate the regular toroldal convolution in the limiter shape. The magnetic field is tangential to the limiter surface at the middle of the bay while at a distance of $\sim 25 \mathrm{~cm}$ from the middle the surface is recessed from the LCFS by $\sim 5 \mathrm{~mm}$. With the magnetic field orientation depicted in Fig. 2 and the limiter shape shown in Fig. 3 , we would expected (if the Cosine is followed) that within a given bay that the interaction would be most intense in the upper left and lower right quadrants as indicated in Fig. 2. In constrast, the upper right and lower left quadrants should produce weak signals since neighbouring bays should shadow these regions.

\section{Comparison of C.II Poloidal Array Distributions with LIM}

In this section we compare $C$ II data taken with the array with predictions from the $L I M$ code. The discharge conditons were the same as in Section $I$, le the major radius $\left(R_{0}=2.45 \mathrm{~m}\right)$, the minor radius $(a=0.80 \mathrm{~m})$, the plasma current $\left(I_{p}=1.4 M A\right)$, the Ohmic power input $\left(P_{\mathrm{\Omega}}=1 \mathrm{MW}\right)$ and the toroidal magnetic field $\left(B_{T}=4 T\right)$. The discharge conditions were varied by changing the density through deut ${ }^{\prime \prime}$ am gas puffing. The range of (volume-averaged) density extended from a lower limit of $\left\langle n_{e}\right\rangle \sim 1 \times 10^{19} \mathrm{~m}^{-3}$ to a high density disruptive limit of $\left\langle n_{e}\right\rangle \sim 4 \times 10^{19} \mathrm{~m}^{-3}$. 
The code input assumptions were the same as in Section I, le background plasma conditions are determined by the Langmuir probes in the SOL and ECE and interferometer measurements in the main plasma.

Figure $4 a, b, c$ show the experimental array $C \|$ distributions, dots, and the LIM code results for C I, C II, C III lines for $\left\langle n_{e}\right\rangle \sim 1.1 \times 10^{19} \mathrm{~m}^{-3}$ (TFTR shot \#45423), $<n_{e}>\sim 2.7 \times 10^{19} \mathrm{~m}^{-3}(\# 45436),<n_{e}>\sim 3.9 \times 10^{19} \mathrm{~m}^{-3}$ (\#45438), Figs. 4a,b,c, respectively.

As can be seen, the agreement between the experimental $C \|$ distribution and the LIM prediction is good in the low density case, Fig. 4a., both for the overall pololdal width of the distribution as well as the signal level at the midplane. The overall pololdal width of the distribution is mainly determined by the radial decay of the particle flux in the SOL. The signal at the tangency point at the midplane, if the Cosine Law were strictly followed, should be zero. However, in this discharge, at low density (Fig. 4a), the finite signal at the midplane can be explained by the mean-free paths of the atoms sputtered from regions above and below the midplane. This tends to fill in the valley at the midplane, as predicted by LIM and observed experimentally.

At higher densities, Figs. 4b,c, the experimental $C$ II poloidal width is reproduced by the LIM code. However, the experimental midplane signal, which should decrease with increasing density if the Cosine Law were followed, owing to reduced mean-free paths, actually increases. The LIM prediction follows the expected trend, le the midplane signal decreases with density. The opposing trends are clearly demonstrated in Fig. 5 for the entire density scan of Section I.

We thus conclude that at moderate to high density significant plasma flux is reaching the midplane region, a region which, if the Cosine Law were followed, should receive little flux. The midplane flux appears to increase in importance with increasing density. At low density, we cannot say whether the Cosine Law is adhered to, since LIM predicts significant mean-free paths for sputtered atoms which results in a significant signal at the midplane.

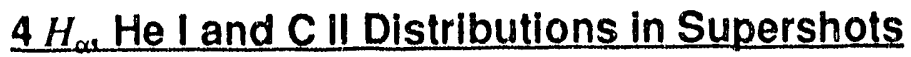

The poloidal array gives only limited spatial resolution for a complicated two-dimensional distribution. We have therefore made use of the periscope viewing system 
on TFTR (Bay $P$ ) and the newly purchased intensifled CCD camera to obtaln two-dimensional images in $H_{\alpha}, \mathrm{He} I$ and $\mathrm{C} I 1$ light. Figures 6,7,8 give the three dimensional intensity plots, as well as corresponding contour maps, for a Supershot plasma with $I_{n}=0.8 M A, B_{T}=3.5 T, R_{0} d a=2.45 \mathrm{~m} / 0.80 \mathrm{~m}, \mathrm{NBI}$ power $P_{N B I} \sim 9 \mathrm{MW}, \Lambda \sim 2.1$, $\bar{n}_{e} \sim 1.9 \times 10^{19} \mathrm{~m}^{-3}$, deuterium beams with hellum tracer gas-puffing.

The spatial distributions of the three emissions are similar. The toroldal modulathon which repeats at each bay is clearly visible. There is some qualitative agreement between these distributions and what would be expected based on the Cosine Law, le we expect the interaction to be most intense at the upper left and lower right quadrant and there is a slight tendency for the experiment to show this. However, in general the distributions are in sharp contradiction to what is expected based on the Cosine Law; for example, (1) The upper right and lower left quadrants should show little signal due to shadowing from nelghbouring bays whereas the observed interaction is much stronger.

(2) The intensity should be a minimum in the middle of the bay, where the fleld is approximately parallel to the surface, instead, a maximum is observed. (3) As with the observation with the poloidal array at Bay $B$, the interaction at the midplane region should be weak whereas experimentally we observe a significant signal.

\section{Two-Dimensional Analytic Modelling}

In this section we compare in detall the two-dlmensional $H_{\alpha}$ pattern with predictions from a simple analytic model in which we first assume the Cosine Law. We will then later demonstrate that only by discarding the Cosine Law can we get reasonable agreement between the experimental results and the predictions of the simple model.

We make the comparison with an Ohmic discharge similar to that from Section 1: $I_{p}=1.4 M A, B_{T}=4.0 T, R_{0} / a=2.45 \mathrm{~m} / 0.80 \mathrm{~m}$, Ohmic power $P_{\mathrm{Q}} \sim 1 \mathrm{MW}, \bar{n}_{\mathrm{e}} \sim 3.0 \times 10^{19} \mathrm{~m}^{-3}$, deuterlum gas-puffing. The experimental $H_{\alpha}$ intensity pattern is shown in Fig. 9. As before, the signal peaks around the middle of the bay $(y=0)$, signiflcant emission originates in the upper right quadrant which should be shadowed, and the midplane signal is large.

In the analytic model the scrape-off layer plasma, as measured with the probe $\left\langle T_{e}(a)=25 \mathrm{eV}, \lambda_{T}=14 \mathrm{~cm}, n_{e}(a)=8 \times 10^{18} \mathrm{~m}^{-3}, \lambda_{n}=4 \mathrm{~cm}, \lambda_{\Gamma}=3 \mathrm{~cm}\right)$, is mapped onto the limiter using the full three-dimensional shape of the limiter (using a CAD program) and the 
calculated two-dlmensional magnetlo geometry. In the main/global scrape-off layer the particle e.folding distance is measured by the Langmuir probe to be $\lambda_{r}=3 \mathrm{~cm}$. Because of the expanslon of the magnetlo flux surfaces the correpsondling value at the limiter is $\lambda_{\Gamma}=5 \mathrm{~cm}$.

The Cosine Law has been used to determine the flux density onto the surface. Results appear in Fig. 10. As expected, the flux density is small at the middle of the bay and the midplane and vintually no flux reaches the upper right quadrant.

In addition to the maln/global scrape-off layer, there is a "prlvate" scrape-off layer that exists between bays on the inner bumper limiter. This reglon arises due to the reg. ular toroldal convolutions in the limiter shape. In the absence of cross-fleld transport of partloles, neighbouring bays should completely shadow portlons of each other. This is only partly observed and thus the above calculation has been repeated in Fig. 11 with the shadow reglon or "private" scrape-off layer having a non-zero particle flux radlal $\theta$-folding distance. We have used a value of $\lambda_{\Gamma}^{\circ}=2 \mathrm{~mm}$, which is approximately consistent with the global value of $\lambda_{\Gamma}=3 \mathrm{~cm}$ when account is taken of the connection lengths, le $30 \mathrm{~cm}$ in the shadow region between bays compared with $70 \mathrm{~m}$ in the global $\mathrm{SOL}$. As can be seen In Fig. 11, the effect of the private SOL is to Increase the Interaction in the otherwise fully shadowed reglons. However, the analytic calculation still produces a minimum in the middle of the bay due to the Cosine Law.

In Figures 12 and 13 are results from the model with the Cosine Law taken out, ie the flux density onto the surface is assumed to be independent of angle and proportion simply to the parallel field flux density as specified by the probe. Fig. 12 assumes full shadowing (no private SOL) while Fig. 13 includes the $2 \mathrm{~mm}$ SOL between bays. The removal of the Cosine Law produces at maximum in the flux density near the middle of the bay, in good agreement with experiment. Note that we still need the private SOL io reproduce the signiffcant levels observed in the otherwise shadowed regions.

It therefore appears that over most of the limiter the particle flux falling on the surface is best described as being independent of the angle of the field line with the surface. Such an observation has also recently been made in an experiment on the DITE tokamak [1], where it was found that for angles $|90-\theta|<3^{\circ}$ the flux was independent of 
angle. Figure 14 shows a scatter plot of $|90-\theta|$ values across the limlter face for the TFTR bumper llmiter. One can see that over most of the surface the angle is typlcally $\sim 1^{\circ}$, in the same range as the DITE experiment.

At angles between the surface normal and the magnetlc fleld of $\theta \sim 89^{\circ}$, the Cosine Law would predict a reduction of the parallel fleld flux density by a factor of $\cos 89^{\circ} \sim .017$. At this small level, the cross-field anomalous particle flux onto the limiter surface starts to become comparable to the reduced parallel flux. The cross-fleld flux is presumably Independent of angle over this range and thus the results are consistent with the orossfleld flux dominating the parallel partlcle flux for most of the limiter surface.

\section{Summary}

(i) The plasma flow pattern to the limiter is observed to be very different than at first expected. It is clear that the usual assumption that the particle flux is reduced by the cosine of the angle $\theta$ between the surface normal and the magnetic field is incorrect. That is, the flux derisity on the surface appears to be independent of fleld angle for most of the TFTR limiter.

(ii) When $|90-\theta|<3^{\circ}$ the parallel-field flux to a surface is reduced to the point where cross-field flux becomes significant and even dominant (as found in [1]).

(iii) A recent paper [2] on the angular dependence of power flow to the divertor plates of DIII does not agree with these findings. The cosine dependence of the power flux is observed. Further work is required to reconcile this discrepancy.

(Iv) Because of the Importance of the cross-field flux to the limiter surface, the wetted area of the limiter is between $50 \%$ and $100 \%$ larger than expected assuming simple parallel flow and full shadowing by adjacent bays. This Implles that the average limiter power loading is lower than expected during high power operation.

\section{ZImplication}

Large, high-power tokamaks have a severe power handling problem at the plasma-surface interface. Typically, $50 \%$ of the input power in machines like TFTR and JET 
is exhausted from the plasma in the form of energetic particles striking relatively small structures llke the limiters or divertor plates. In ITER the exhaust power reaching the SOL is expected to be $\sim 100 \mathrm{MW}$. It is normally assumed in the design of these firstwall structures that the density of exhausted particles and power striking the surface can be reduced to arbitrarily low values by making he angle between the fleld and the surface very glancing. A tentative conclusion from this work is that this arbitrary reductlon may not be possible at very glancing angles, that is, where $|90-\theta|<3^{\circ}$. This may have very serious impllcations for the designs of future machines, for example, ITER.

\section{References}

(1) G F Matthews et al, Plas Phys Contr Fus 32 (1990) 1301.

(2) G F Matthews et al, Nucl Fus 31 (1991) 1383. 


\section{Figure Captions}

(1) Camera viewing geometry on TFTR.

(2) The field of view of the Bay P camera on TFTR looking at the inner bumper limiter.

(3) Toroidal profile of the limiter surface at the mid-plane compared with the last closed magnetic flux surface.

(4) Comparison of the experimental poloidal C II distribution with results from the LIM simulation for C I, C II and C III: (a) low density (b) moderate density (c) high densiiy.

(5) Comparison of experimental and LIM predictions of the $\mathrm{C} I \mathrm{~m}$ midplane intensity to peak intensity as a function of plasma density.

(6) $\quad H_{\alpha}$ intensity distribution on the TFTR bumper limiter in Bay $\mathrm{P}(\mathrm{a})$ three-dimensional plot (b) intensity contour plot.

(7) He l intensity distribution on the TFTR bumper limiter in Bay $P$ (a) three-dimensional plot (b) intensity contour plot.

(8) C Il intensity distribution on the TFTR bumper limiter in Bay P (a) three-dimensional plot (b) intensity contour plot.

(9) Experimental $H_{\alpha}$ intensity pattern above the mid-plane for Bay P.

(10) Model prediction for the particle flux density to the TFTR bumper limiter assuming the Cosine Law and no particle flux in the shadow regions.

(11) Model prediction for the particle flux density to the TFTR bumper limiter assuming the Cosine Law and particle flux in the shadow regions.

(12) Model prediction for the particle flux density to the TFTR bumper limiter not assuming the Cosine Law and with no particle flux in the shadow regions.

(13) Model prediction for the particle flux density to the TFTR bumper limiter not assuming the Cosine Law and with particle flux in the shadow regions. 
(14) Angle of the limiter surface with the magnetic field as a function of horizontal position on the limiter. 


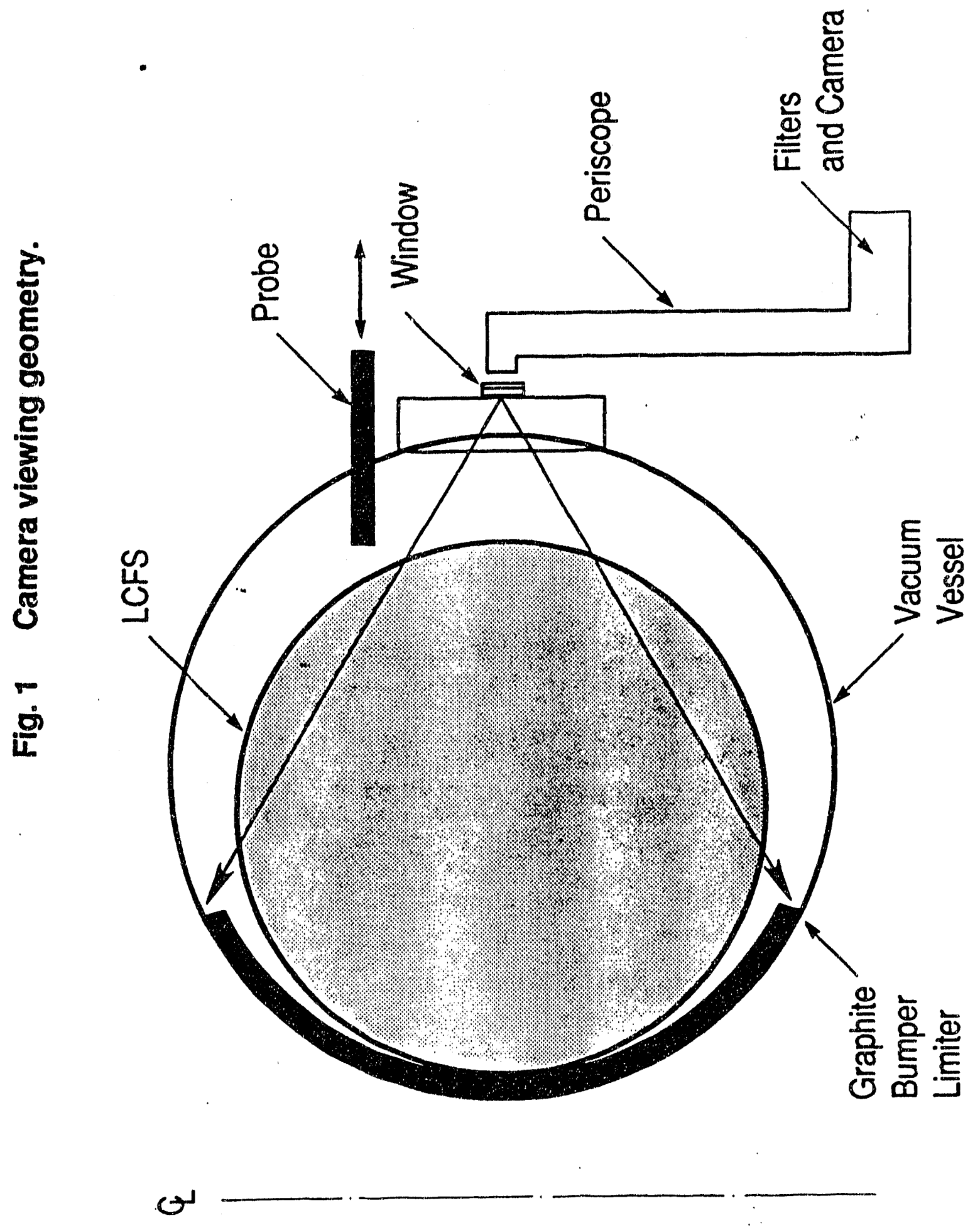




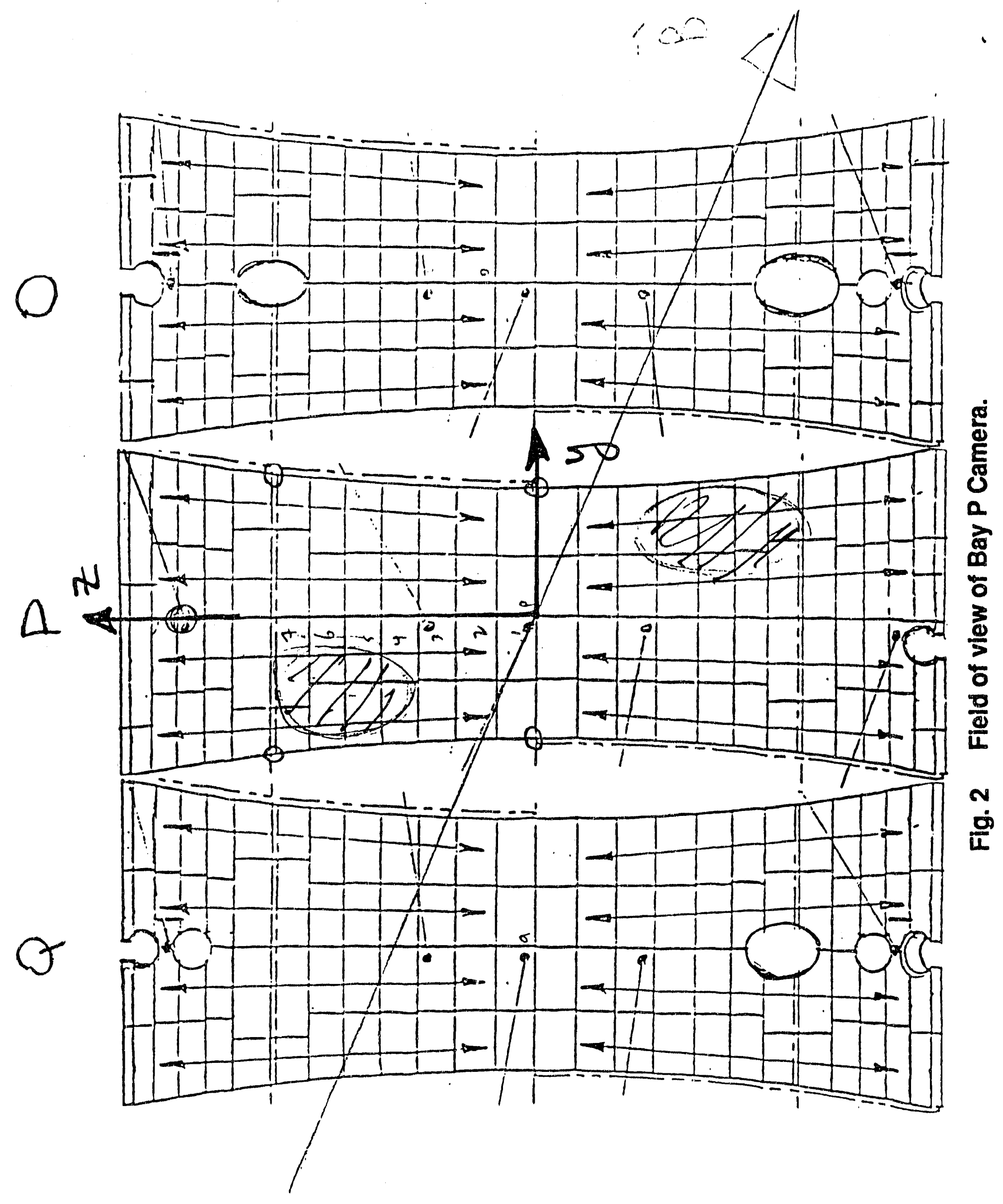




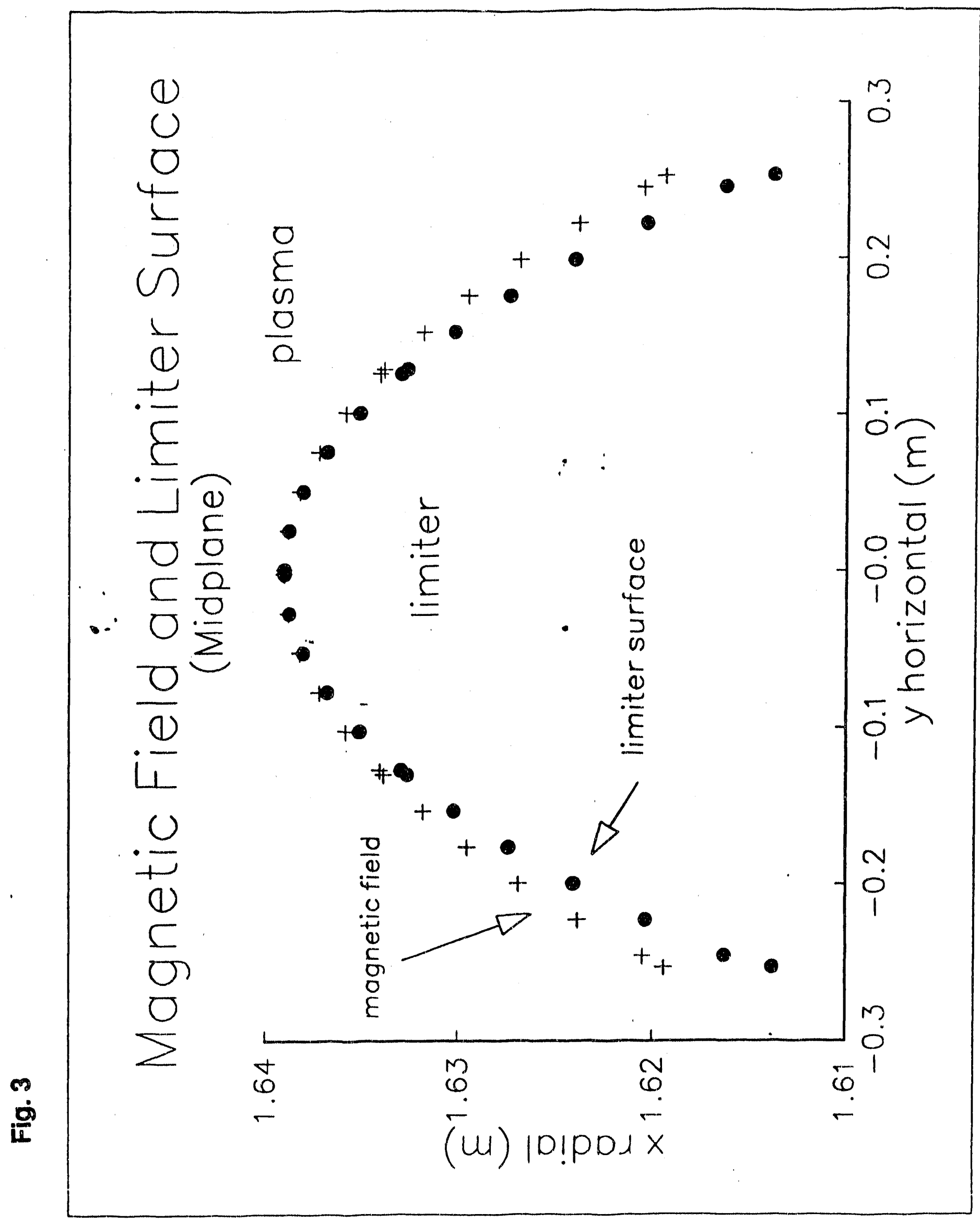


Fig. 4a LIM Simulation

low density $\left\langle n_{e}\right\rangle=1.1 \times 10^{19} \mathrm{~m}^{-3}$, parallel transport only

$C_{C}(909 \mathrm{~nm}) \ldots$ CII $(657 \mathrm{~nm}) \ldots C$ III $(464 \mathrm{~nm})$

- experimental C II (657 nm)

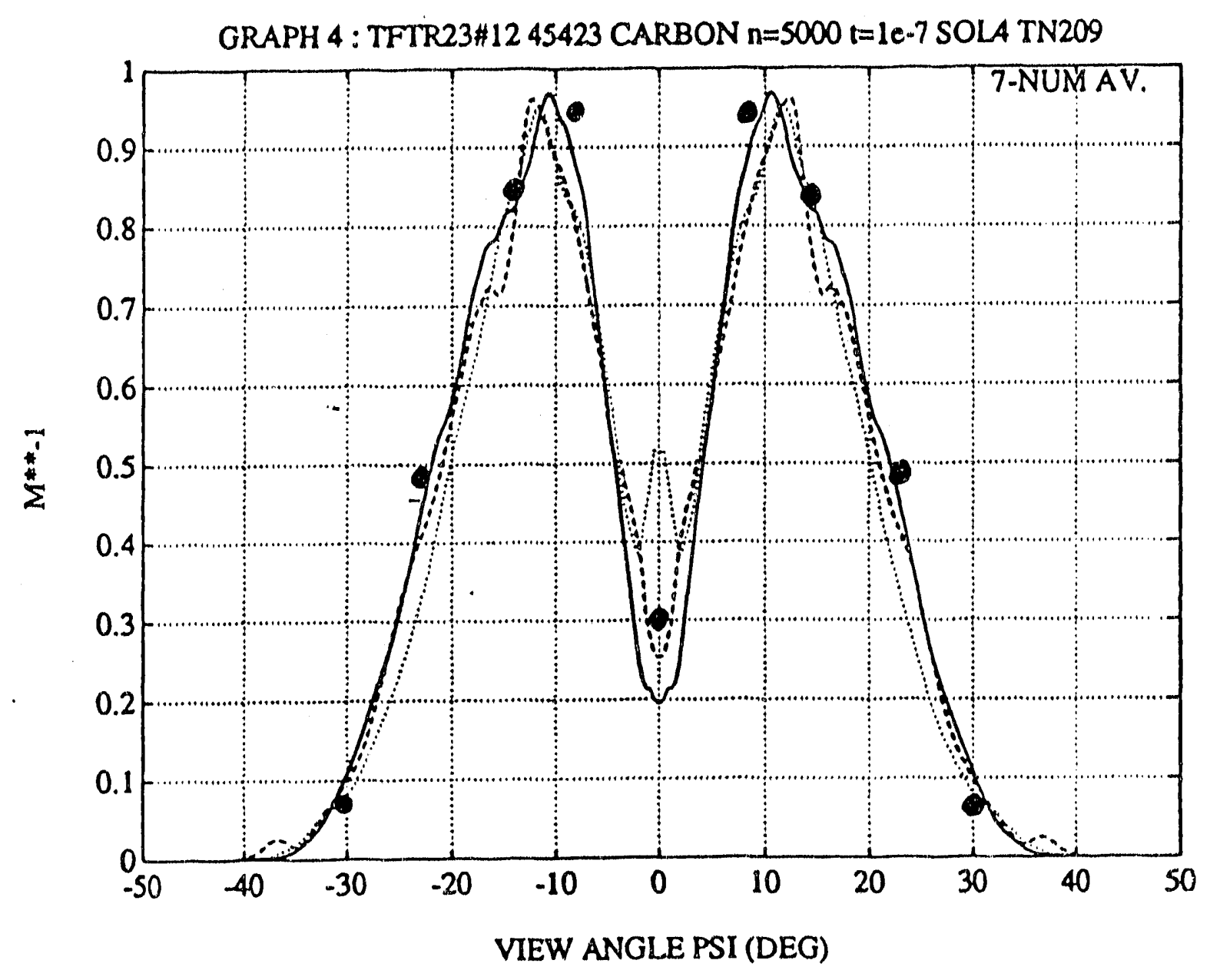


Fig. 4b LIM Simulation

moderate density $\left\langle n_{e}\right\rangle=2.7 \times 10^{19} \mathrm{~m}^{-3}$, parallel transport only

$$
\begin{gathered}
\text { C I (909 nm) _ C II }(657 \mathrm{~nm}) \ldots \text { C III }(464 \mathrm{~nm}) \\
\text { •experimental C II }(657 \mathrm{~nm})
\end{gathered}
$$

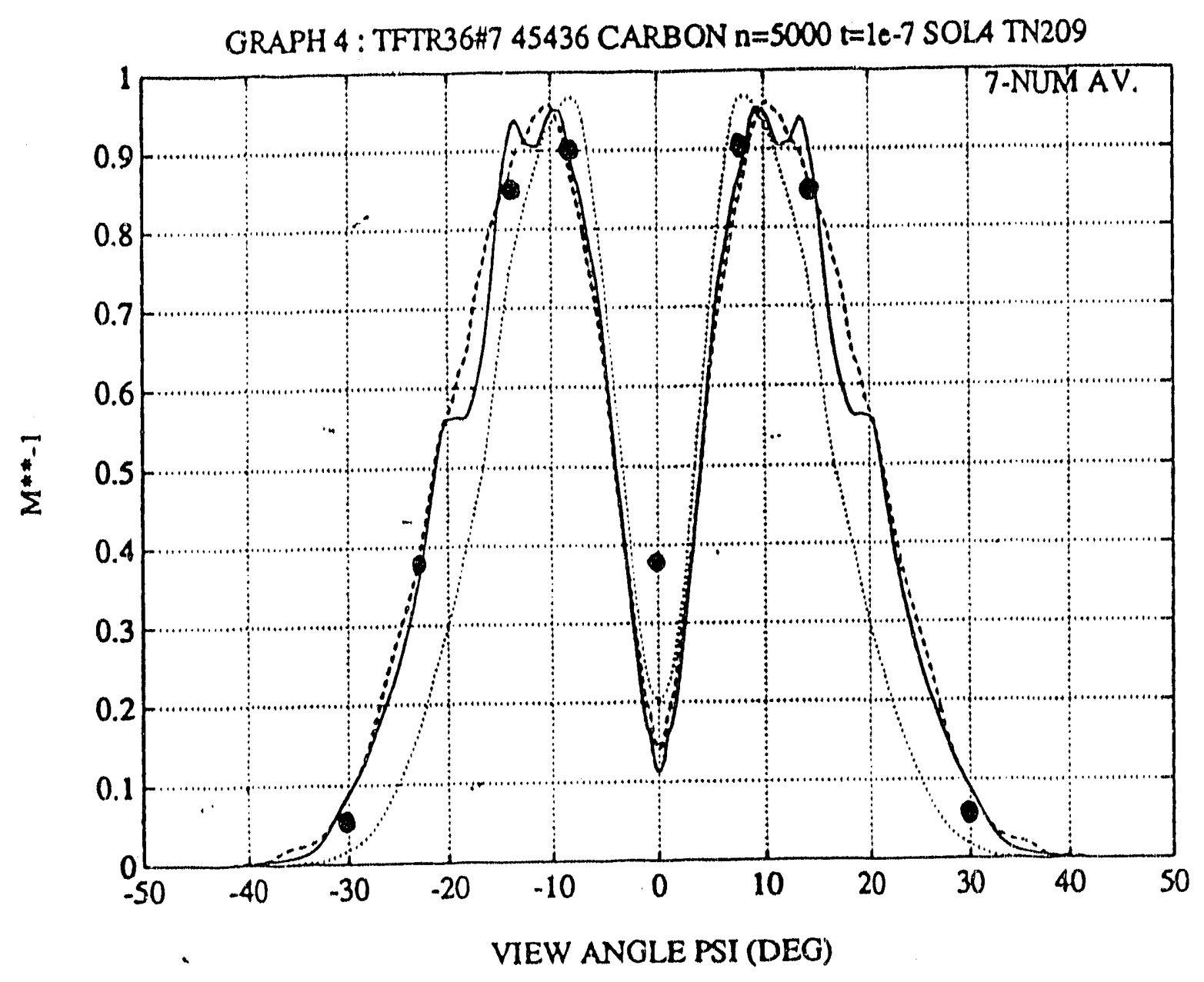


Fig. 4c LIM Simulation

high density $\left\langle n_{c}\right\rangle=3.9 \times 10^{19} \mathrm{~m}^{-3}$, parallel transport only

$\ldots$ CI $(909 \mathrm{~nm}) \ldots$ C II $(657 \mathrm{~nm}) \ldots$ C III $(464 \mathrm{~nm})$

- experimental C II (657 nm)

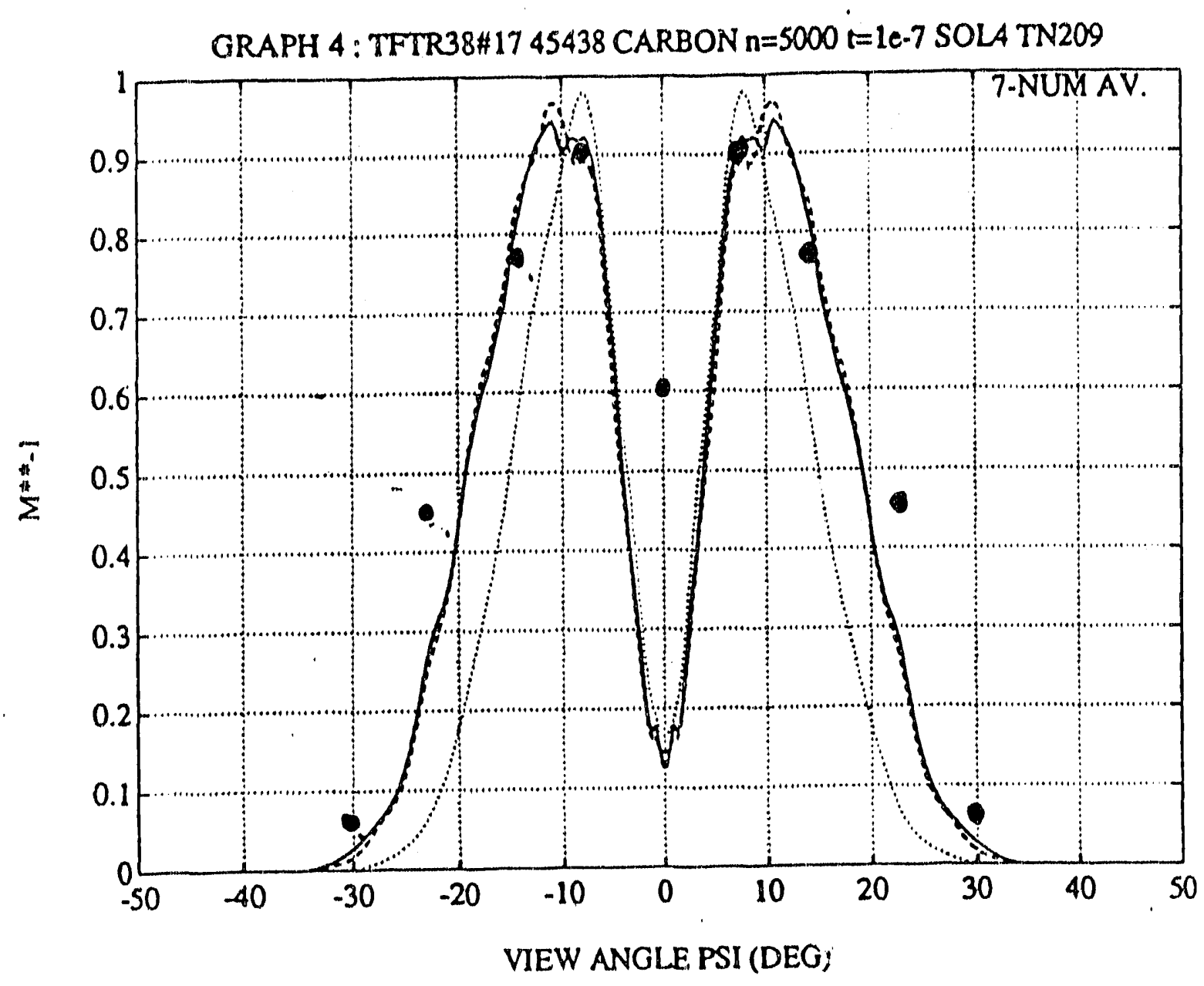




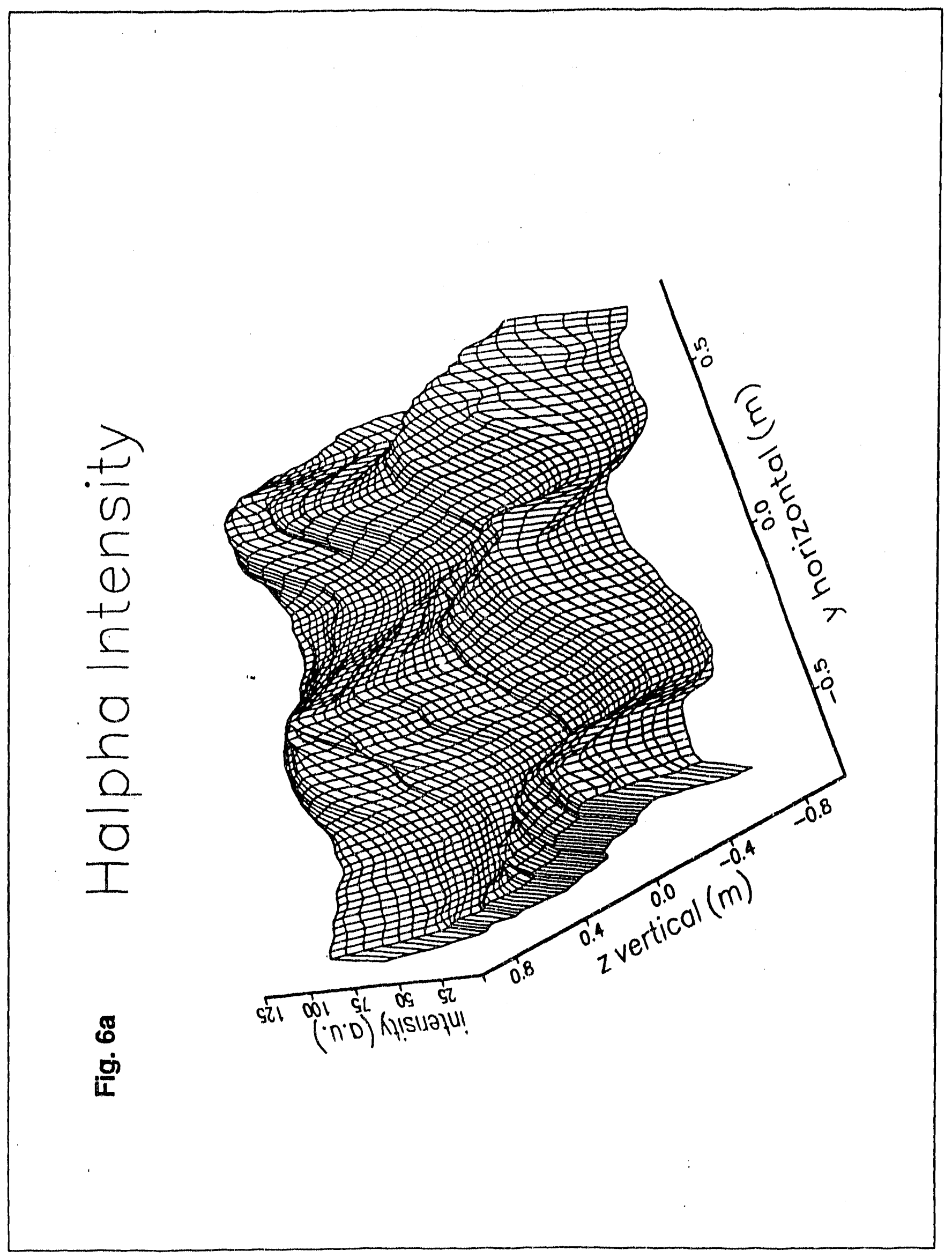




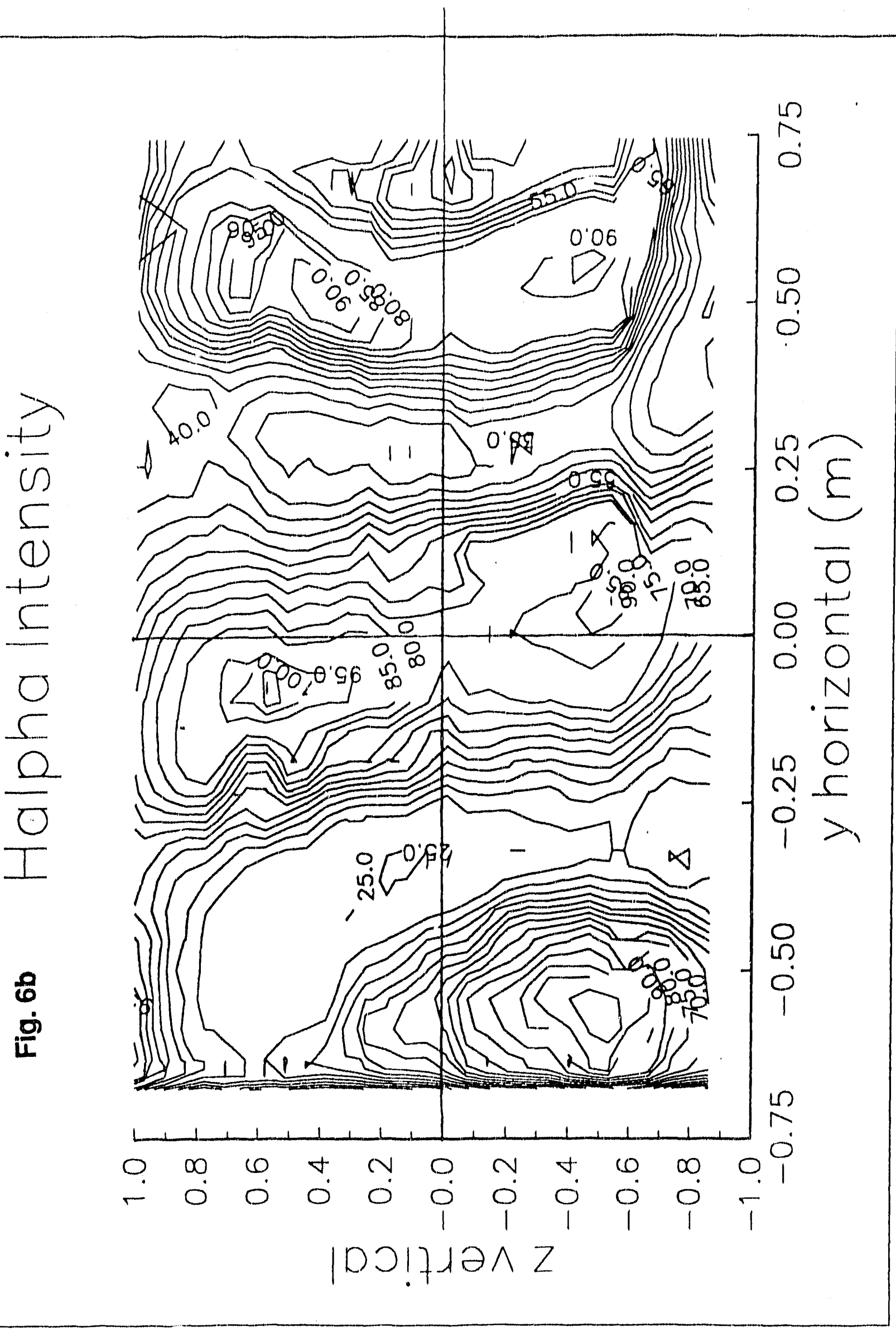




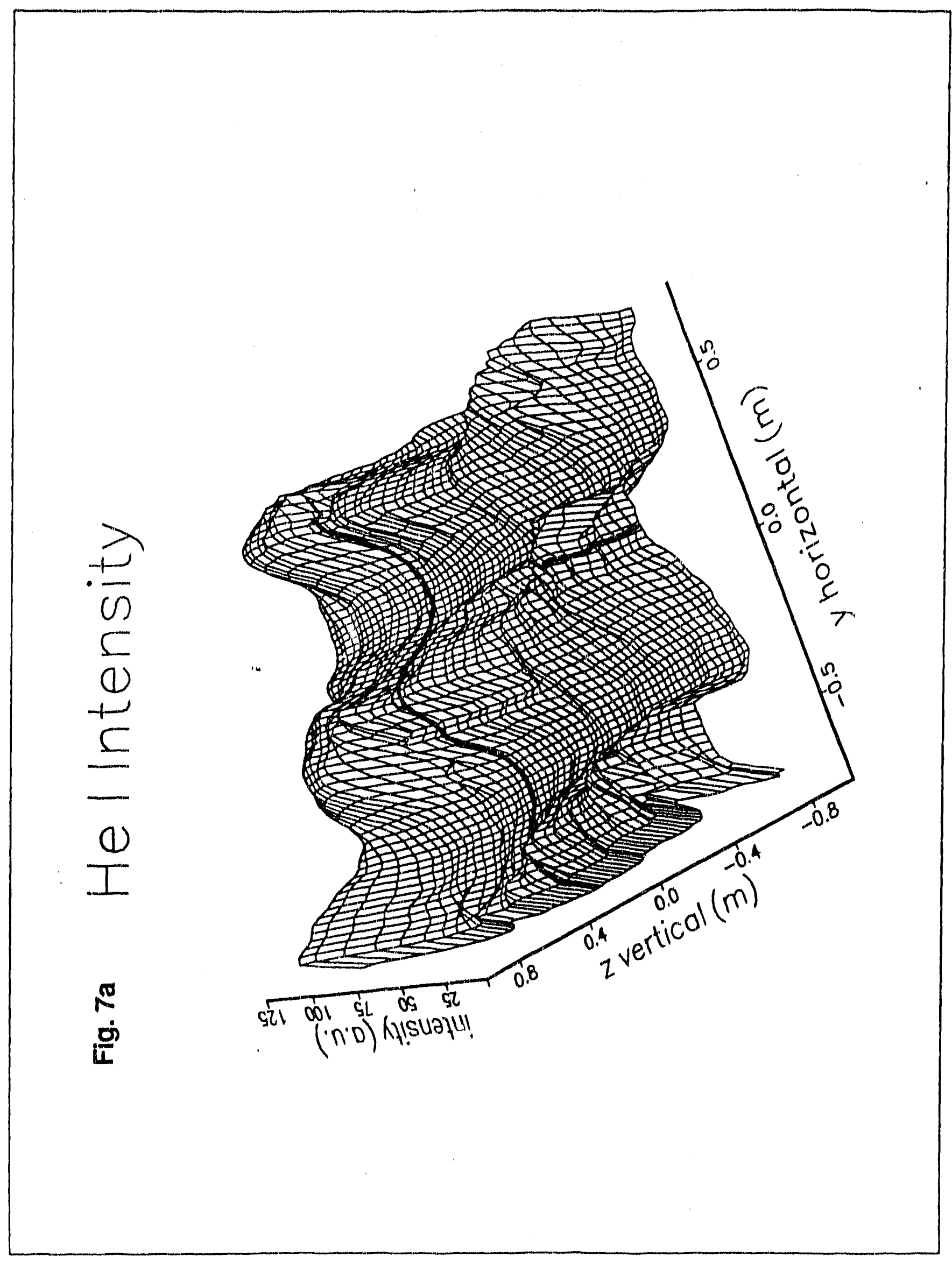




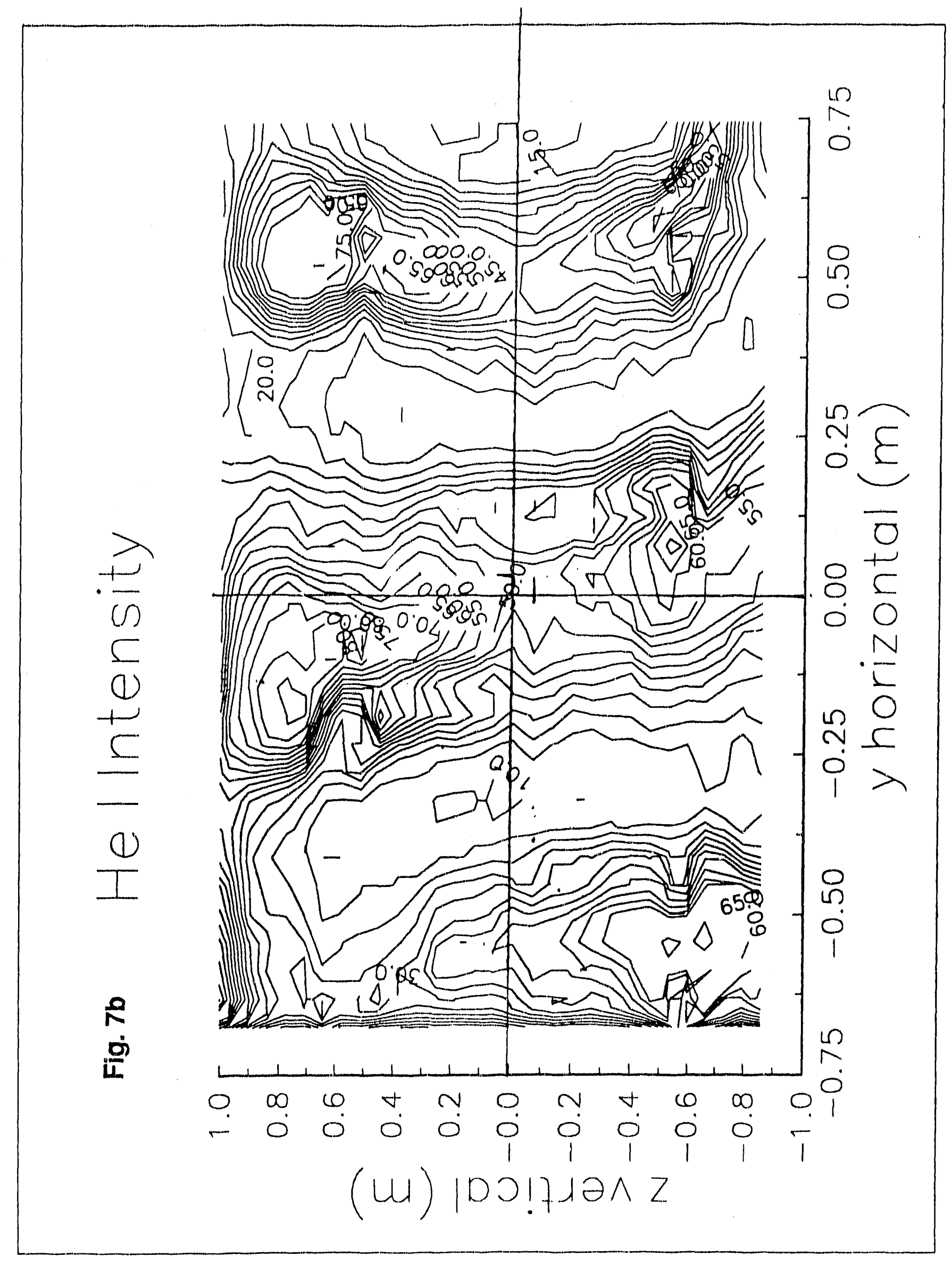




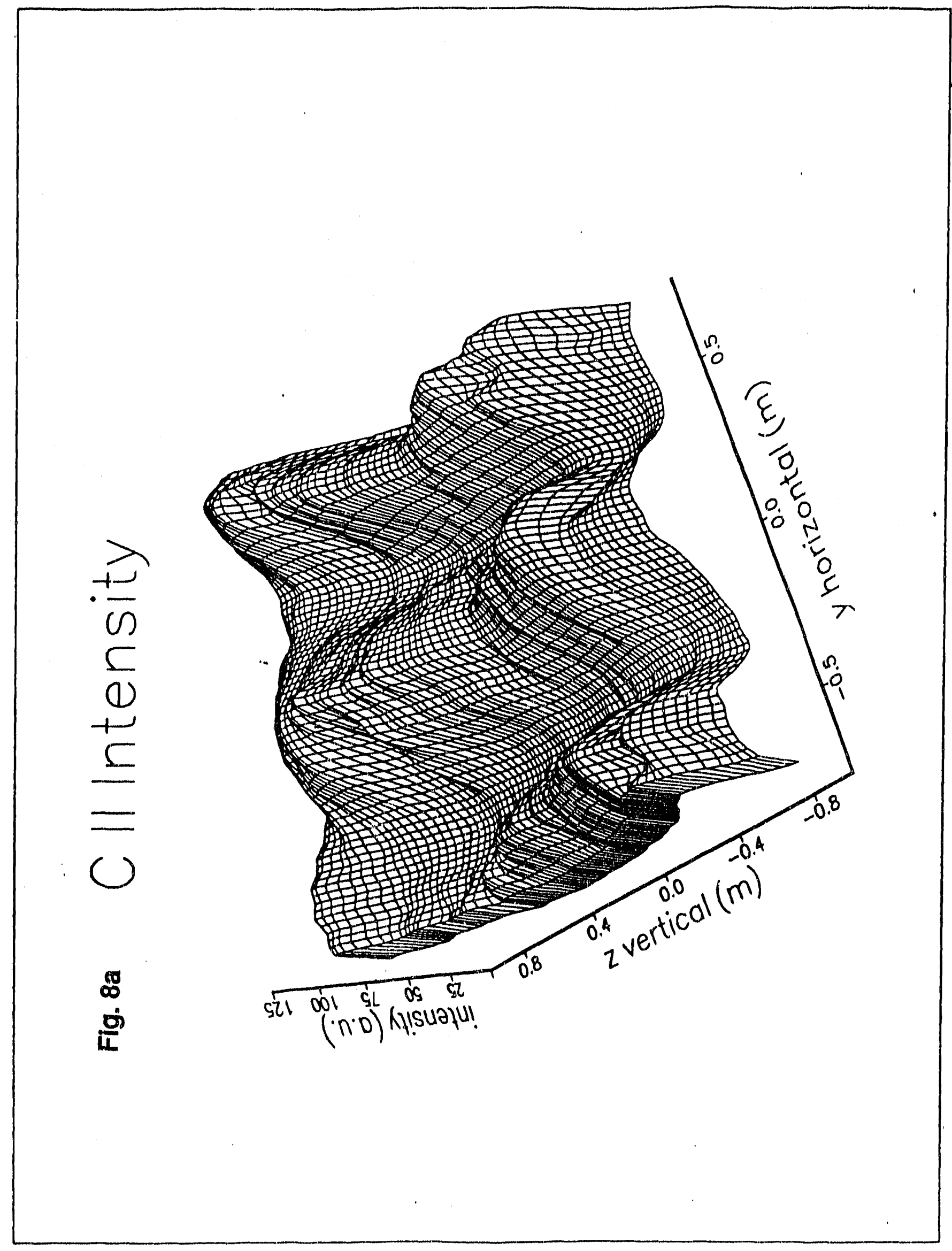




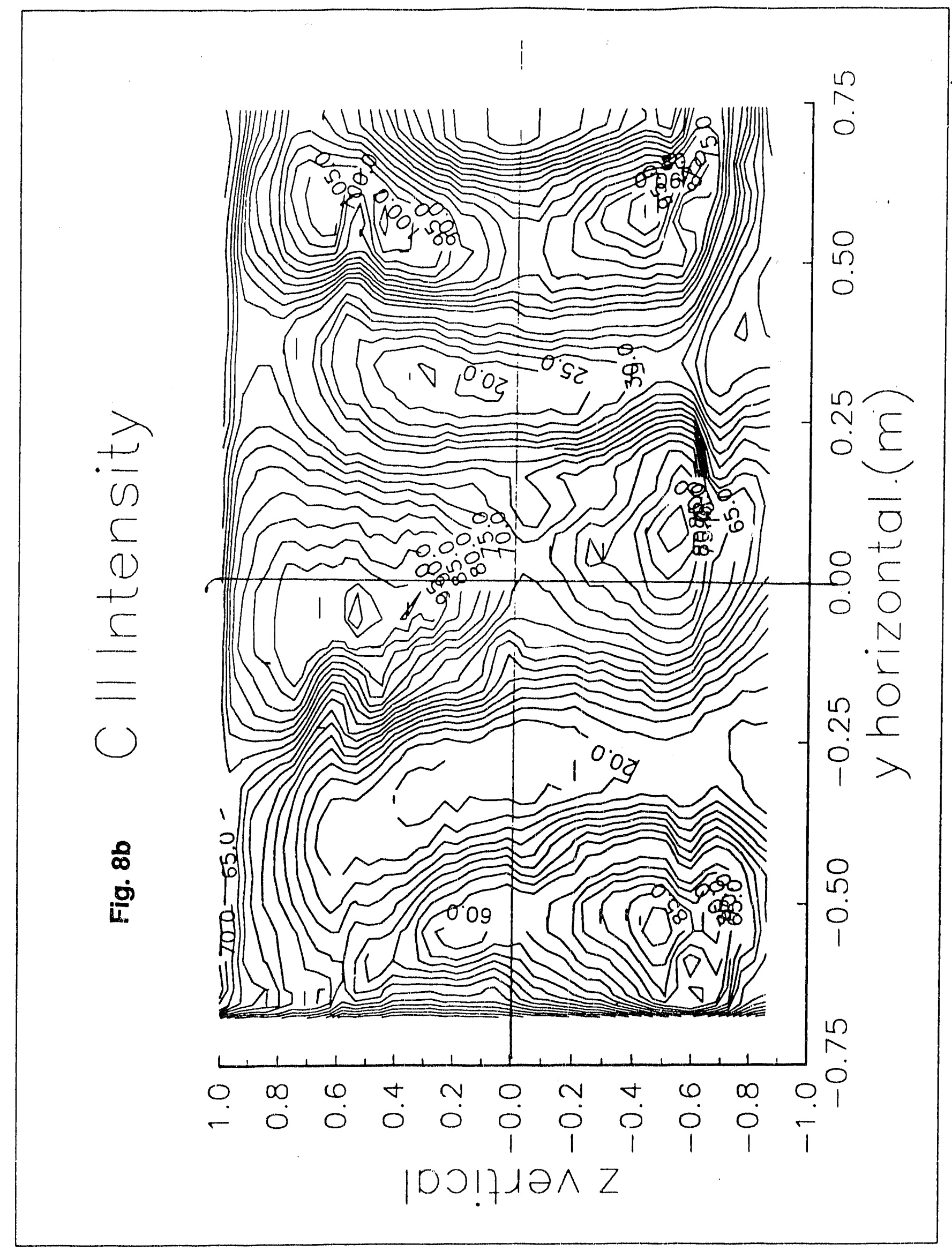




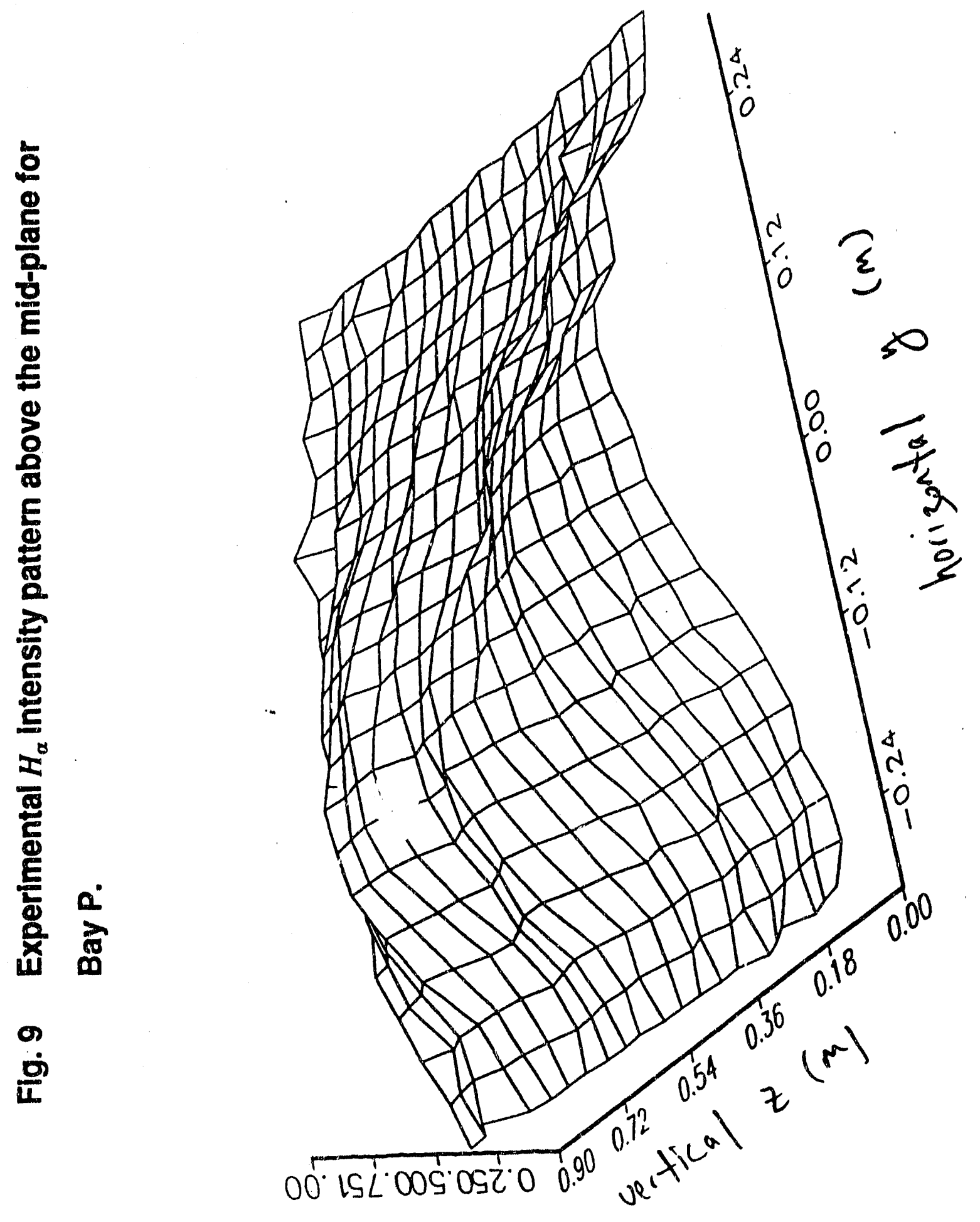




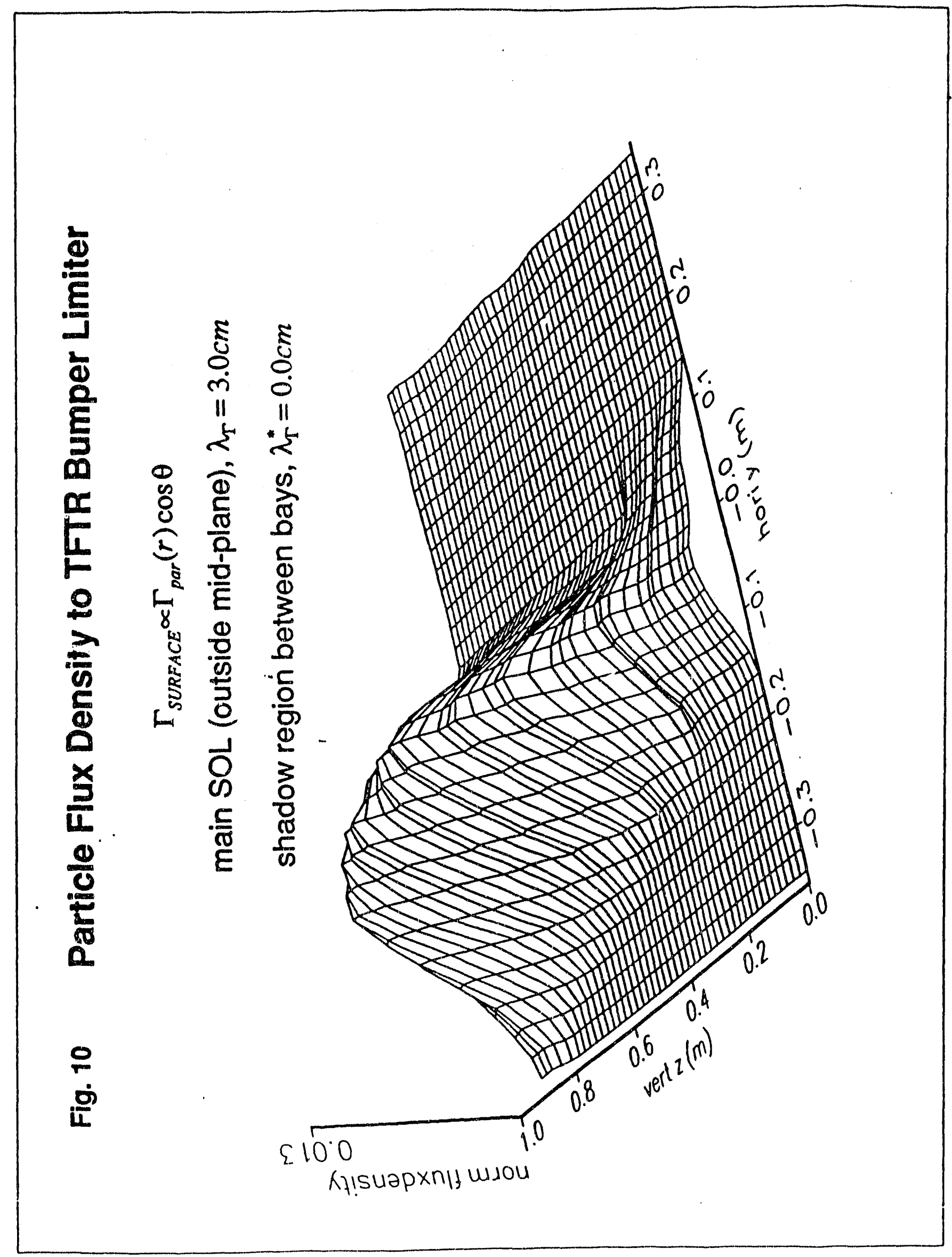




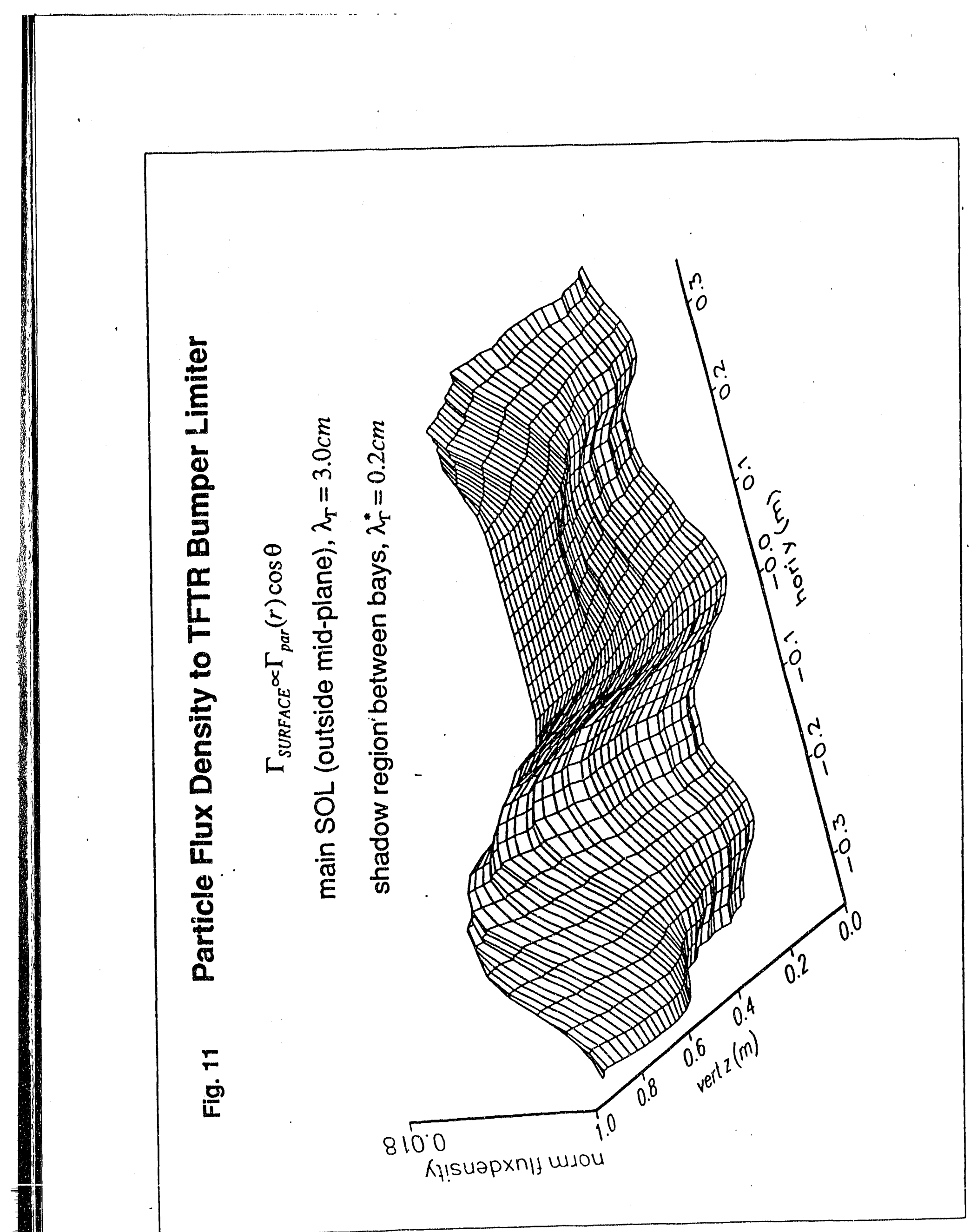




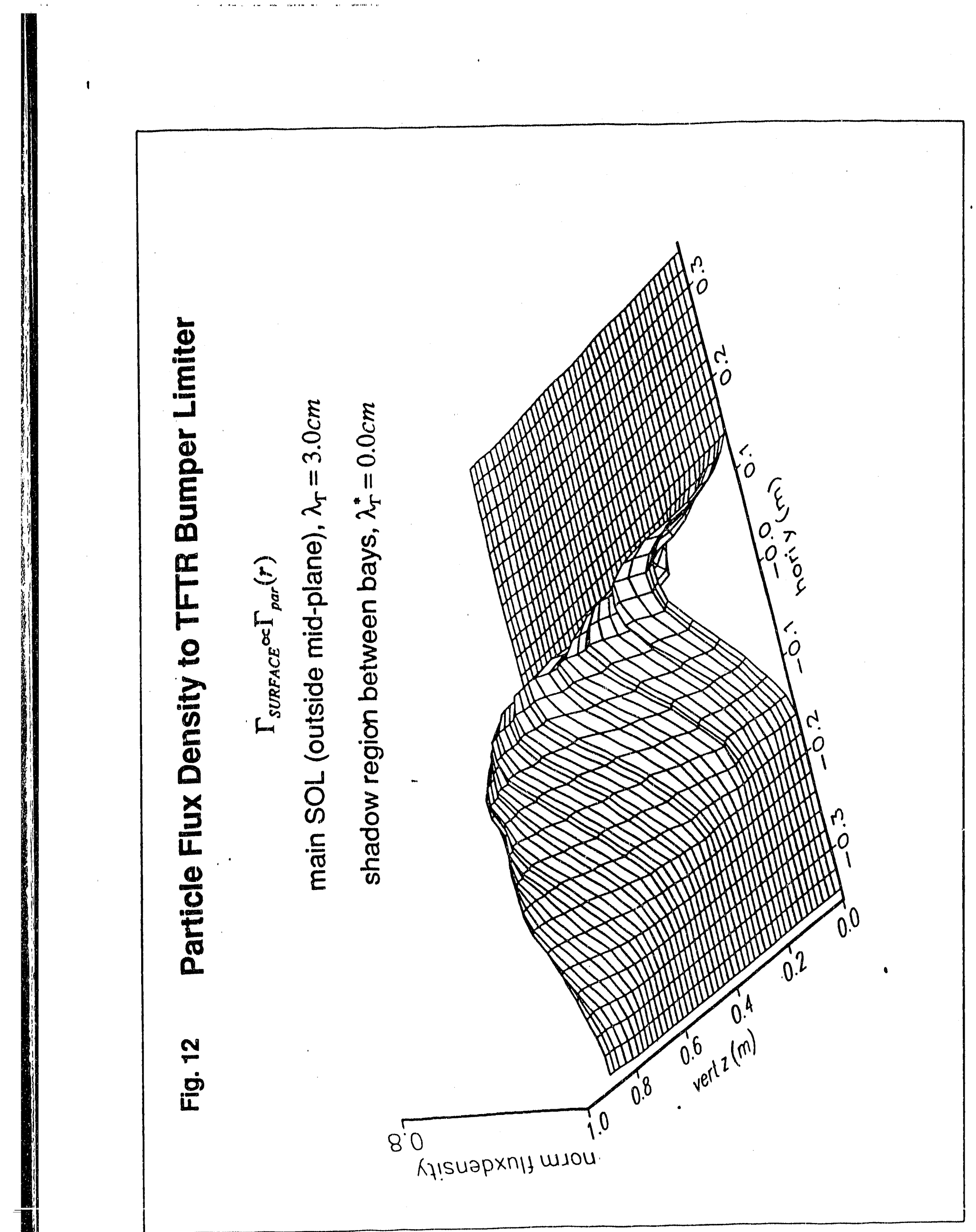




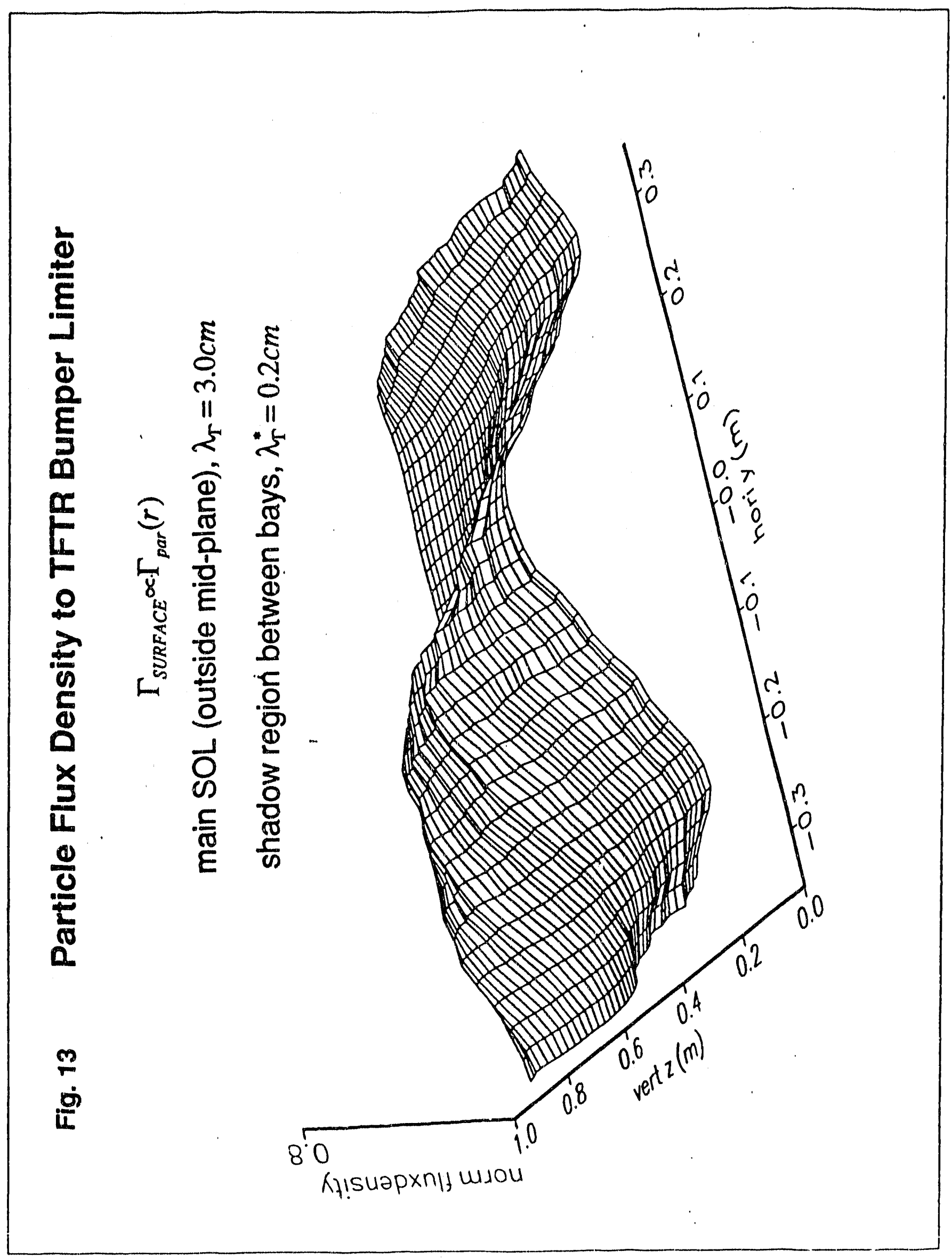


0
0
0
4
5
0
5
$\pm \frac{5}{3}$
0
4
0
0
$\frac{1}{0}$
5

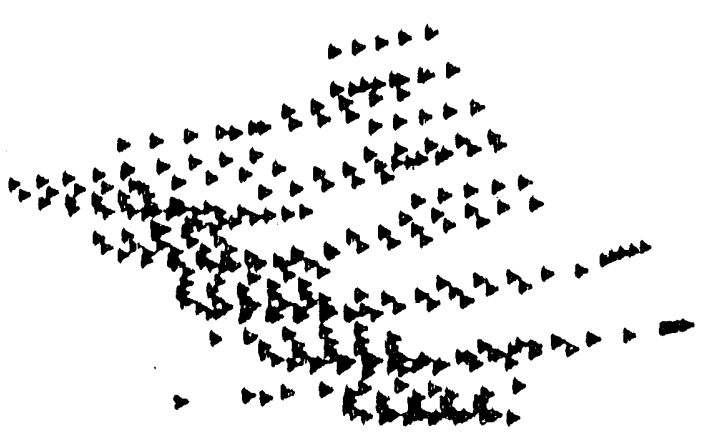

$+$

$M$

0

N

o

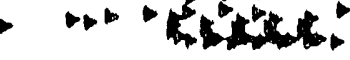

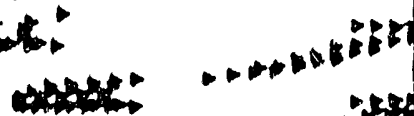

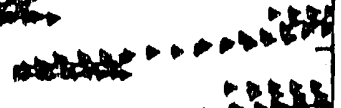

5

- estanos

$\frac{E}{0}$
0.5
$\frac{5}{5}$

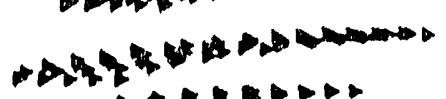

5

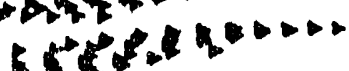

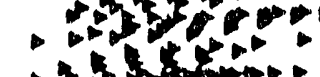

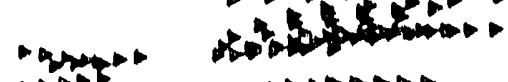

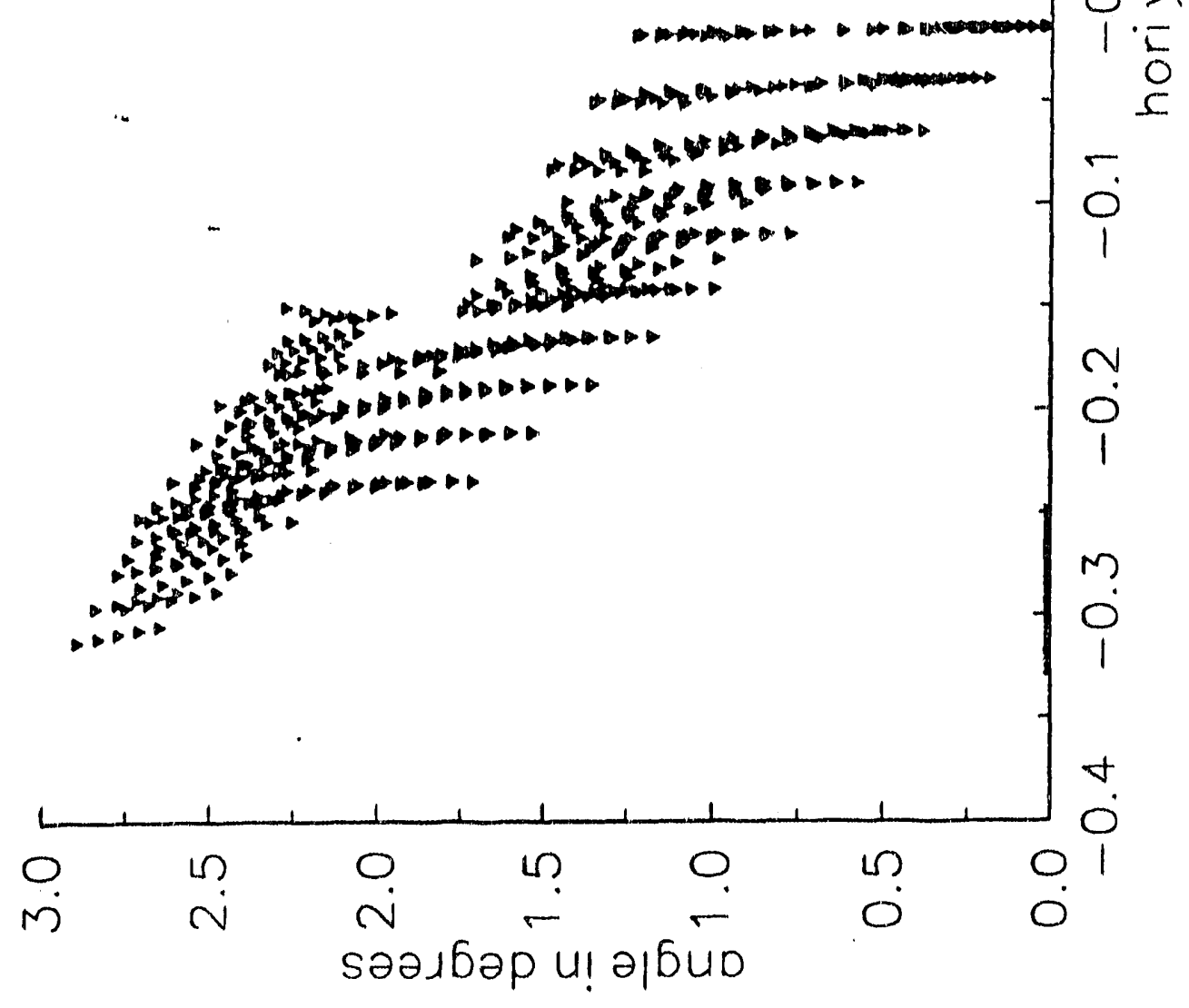




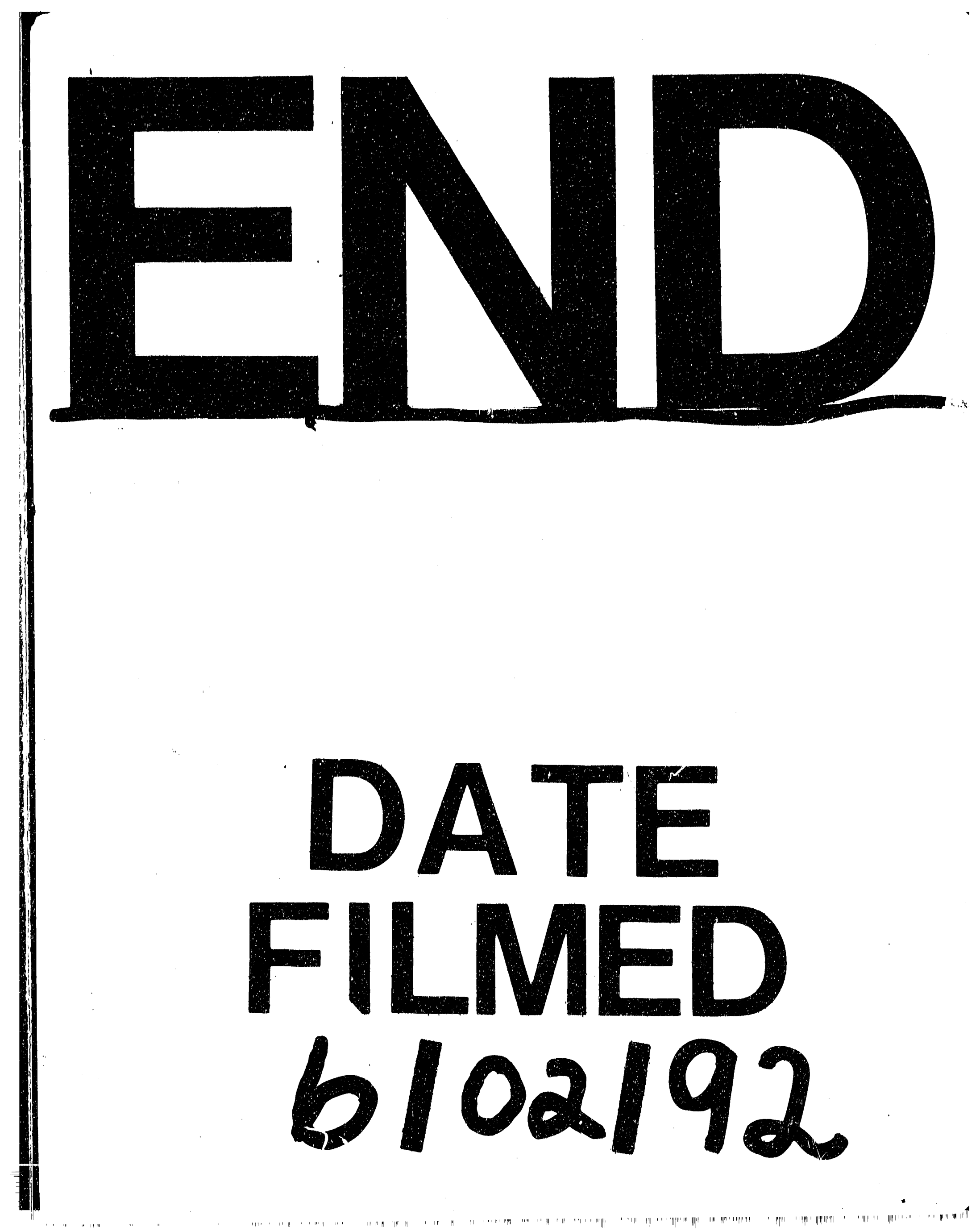


